\title{
Asymmetric Synthesis of Tetrahydrobenzofurans and Annulated Dihydropyrans via Cooperative One-Pot Organo- and Silver-Catalysis
}

\author{
Uğur Kaya ${ }^{\mathrm{a}}$ \\ Pankaj Chauhan ${ }^{a}$ \\ Kristina Deckers ${ }^{a}$ \\ Rakesh Puttreddy \\ Kari Rissanen ${ }^{\mathrm{b}}$ \\ Gerhard Raabe ${ }^{a}$ \\ Dieter Enders*a \\ a Institute of Organic Chemistry, RWTH Aachen University, \\ Landoltweg 1, 52074 Aachen, Germany \\ enders@rwth-aachen.de \\ b Department of Chemistry, Nanoscience Center, University \\ of Jyvaskyla, 40014 JYU, Finland \\ In memory of Professor Jean Normant
}
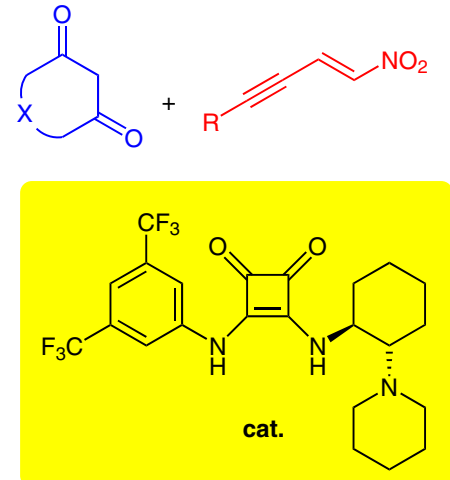

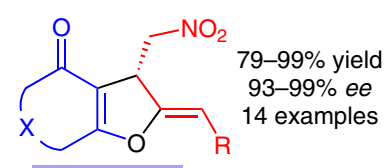

5-exo-dig<smiles>[X]CC1=C(C(=O)CC)C(C[N+](=O)[O-])C=C([R])O1</smiles>

$31-94 \%$ yield 88-94\% ee 3 examples

\author{
Received: 29.04.2016 \\ Accepted: 02.05.2016 \\ Published online: 23.06 .2016 \\ DOI: 10.1055/s-0035-1561468; Art ID: ss-2016-z0305-op \\ License terms: (c) (i) $(\$)$
}

Abstract A low catalyst loading of a squaramide $(0.5 \mathrm{~mol} \%$ ) and a silver(I) salt ( $1 \mathrm{~mol} \%$ ) efficiently catalyzes a one-pot asymmetric Michael addition/hydroalkoxylation reaction between 1,3-diketones and alkynetethered nitroalkenes. Depending on the 1,3-dicarbonyl substrate this cooperative catalytic approach opens access to tetrahydrobenzofurans or annulated dihydropyrans in moderate to excellent yields and very good to excellent enantioselectivities.

Key words asymmetric synthesis, organocatalysis, one-pot synthesis, silver catalysis, annulation

Benzofuran and its partially hydrogenated analogues are important heterocyclic building blocks and very common structures in natural products with interesting biological and pharmaceutical properties. This is also true for structurally isomeric annulated dihydropyrans. ${ }^{1}$ Natural products such as the furanomonoterpene evodone (I), which has been isolated from Evodia hortensis, exhibits significant inhibitory activity on the seed germination of certain species. ${ }^{2}$ Curzerenone (II) and bisabolangelone (III) are other natural products with antibacterial and anti-inflammatory activities, ${ }^{3,4}$ respectively, whereas the diterpenoid maoecrystal V $(\mathbf{I V})$ is a potent selective HeLa cell inhibitor. ${ }^{5}$ The dihydropyran-type natural product crolibulin $(\mathbf{V})$ and the pharmaceutical HA14-1 (VI) show anticancer properties (Figure 1$)^{6}$

Recently, much effort has been invested in the synthesis of tetrahydrobenzofuran and dihydropyran core structures. $^{7}$ Singh and co-workers developed a silver-catalyzed

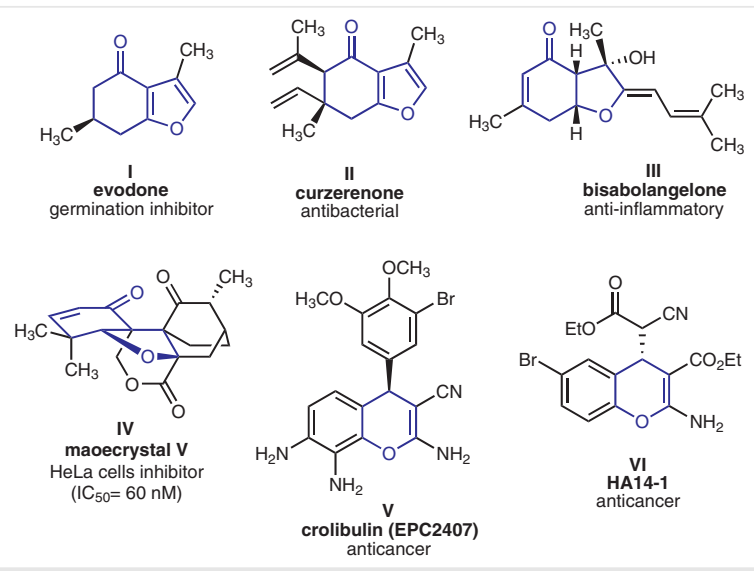

Figure 1 Bioactive natural products and pharmaceuticals containing the partially hydrogenated benzofuran and dihydropyran moieties

interrupted Feist-Bénary reaction between ynones and $\beta$ diketones to provide dihydrofurans in moderate to good yields and good to excellent enantioselectivities (Scheme 1). ${ }^{8}$ Feng and co-workers reported an asymmetric domino Michael addition/O-alkylation reaction between cyclohexane-1,3-dione derivatives and bromonitrostyrenes catalyzed by a bifunctional $N, N^{\prime}$-dioxide organocatalyst to afford polysubstituted bicyclic dihydrofurans. ${ }^{9}$ Calter's group published another interesting synthesis of highly substituted furanoids via an organocatalytic asymmetric aldol/oxaMichael addition sequence between 2-ene-1,4-diketones and dimedone in the presence of a bis(cinchona alkaloid)pyrimidine catalyst. ${ }^{10}$ The Schneider group developed an interesting enantioselective phosphoric acid-catalyzed syn- 
thesis of 4-aryl-4H-chromenes via a conjugate addition of 1,3-diketones to in situ generated ortho-quinone methides followed by a cyclodehydration reaction. ${ }^{11}$

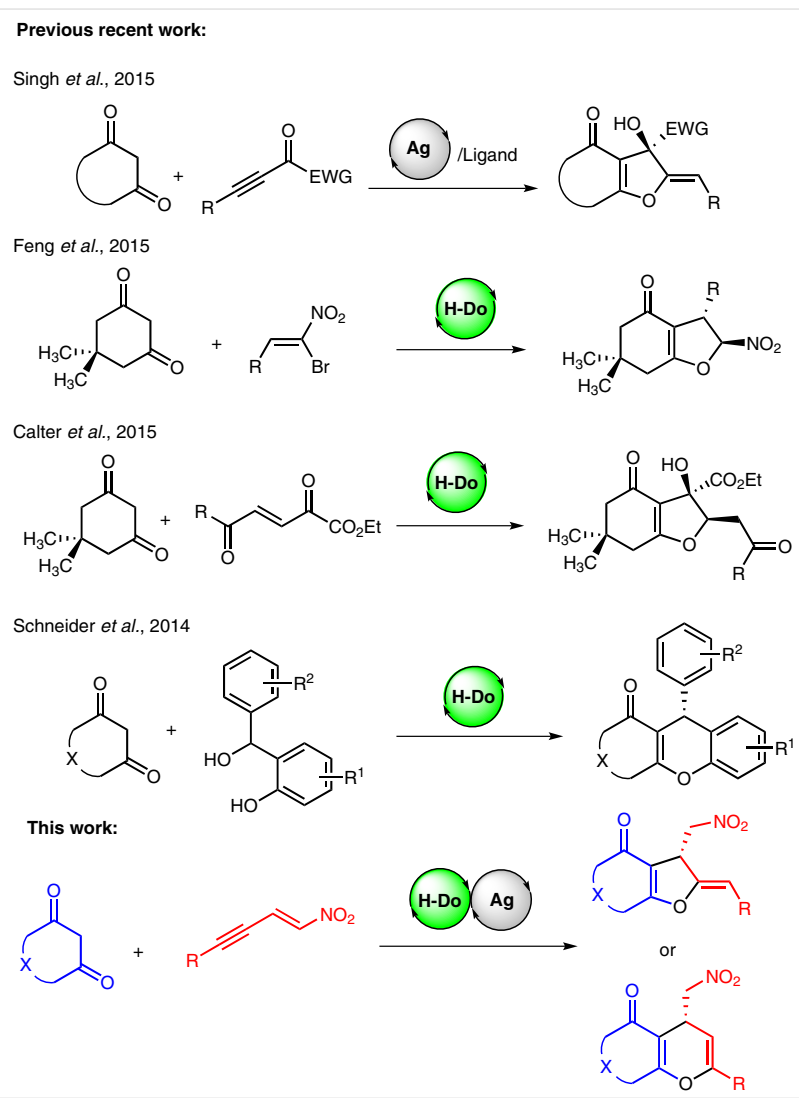

Scheme 1 Approaches for the asymmetric synthesis of tetrahydrobenzofurans and annulated dihydropyran derivatives

The activation of alkynes for subsequent transformations has become an important tool in organic chemistry to develop new and valuable reactions. Alkyne functionalization can be achieved in two crucial routes: $\sigma$-activation ( $\sigma-$ bond metathesis or $\sigma$-coordination) and $\pi$-activation ( $\pi$ complex formation) ${ }^{12}$ The coinage metals $(\mathrm{Cu}, \mathrm{Ag}$ and $\mathrm{Au})$ are suitable candidates for alkyne functionalization due to their good alkynophilicity. ${ }^{13}$ Especially, silver(I) salts have emerged as powerful activators of alkynes. The advantages of stability, nontoxicity, low price, or catalyst compatibility with organocatalysts favor the choice of silver in $\mathrm{C} \equiv \mathrm{C}$ bond activation reactions such as alkynylation, cycloaddition, cycloisomerization, or hydrofunctionalization. ${ }^{14}$

Merging organocatalysis and metal catalysis enables multiple unique transformations in one-pot and this catalytic approach has become a powerful strategy in asymmetric synthesis. Particularly cooperative, relay, synergistic, and dual catalysis variations, where all reactants and catalysts are present from the beginning, is challenging and require high compatibility of the combined catalysts. ${ }^{15}$
In search of new methods for acquiring valuable bioactive heterocyclic compounds and our interest in the combination of organocatalysts and silver(I) salts, ${ }^{16}$ we investigated an asymmetric Michael addition/hydroalkoxylation sequence between 1,3-diketones and alkyne-tethered nitroalkenes catalyzed by a bifunctional squaramide ${ }^{17}$ and a silver(I) salt to provide the desired tetrahydrobenzofurans. We began our investigation by choosing dimedone (1) and nitroalkene $\mathbf{2 a}$ as model substrates. To our delight, the onepot reaction of $\mathbf{1}$ and $\mathbf{2 a}$ in $\mathrm{CH}_{2} \mathrm{Cl}_{2}$ at room temperature catalyzed by squaramide $\mathbf{A}$ and $\mathrm{Ag}_{2} \mathrm{O}$ afforded the desired 5exo-dig cyclization product 3a in $98 \%$ yield and $94 \%$ enantiomeric excess (Scheme 2). Inspired by these excellent results, the reaction was carried out with different squaramide and thiourea catalysts $\mathbf{A}-\mathbf{I}$ along with $\mathrm{Ag}_{2} \mathrm{O}$ as silver(I) salt. All squaramide catalysts as well as thiourea catalysts provided the tetrahydrobenzofuran in high yields and moderate to very good enantioselectivities. The best result was obtained with squaramide $\mathbf{A}$, which gave $98 \%$ yield and $94 \%$ ee.

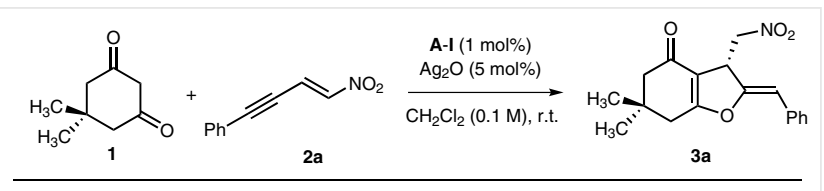<smiles>CN(C)C1CCCCC1NC(=S)Nc1cc(C(F)(F)F)cc(C(F)(F)F)c1</smiles>

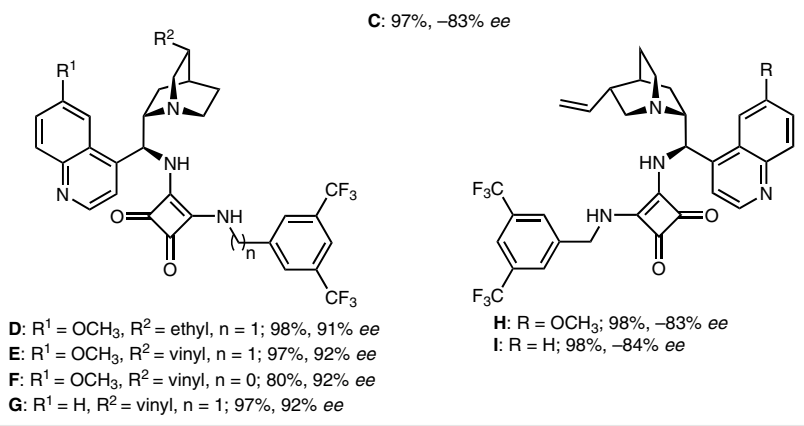

Scheme 2 Catalyst screening for the Michael addition/hydroalkoxylation reaction of $\mathbf{1}$ with $\mathbf{2 a}$

The reaction conditions were optimized further by varying the solvent (Table 1 ). The solvent screening indicated that the chlorinated solvents and $\mathrm{Et}_{2} \mathrm{O}$ gave very good results. The best yields were obtained with $\mathrm{CH}_{2} \mathrm{Cl}_{2}$ and $\mathrm{CHCl}_{3}$. We chose $\mathrm{CH}_{2} \mathrm{Cl}_{2}$ over $\mathrm{CHCl}_{3}$ on the basis of its lower toxici- 
ty. Further optimization studies were carried out by screening transition metal catalysts for the hydroalkoxylation reaction. $\mathrm{Ag}_{2} \mathrm{CO}_{3}$ provided the annulated product with $99 \%$ yield and $95 \%$ ee. The cost aspect led to our decision to use $\mathrm{Ag}_{2} \mathrm{O}$ instead of $\mathrm{Ag}_{2} \mathrm{CO}_{3}$. After carrying out the reaction at different temperatures and catalyst loadings of the squaramide and the silver(I) salt, we determined the optimal reaction conditions, these being $0.5 \mathrm{~mol} \%$ of the squaramide $\mathbf{A}$, $1 \mathrm{~mol} \%$ of $\mathrm{Ag}_{2} \mathrm{O}$, and $\mathrm{CH}_{2} \mathrm{Cl}_{2}$ as solvent at room temperature.

Table 1 Further Optimization Studies ${ }^{\mathrm{a}}$

\begin{tabular}{|c|c|c|c|c|}
\hline${ }_{3} \mathrm{C}^{\prime}$ & & $\begin{array}{c}\begin{array}{c}\text { cat. A } \\
\text { M-cat. }\end{array} \\
\text { solvent }\end{array}$ & $\begin{array}{l}\mathrm{H}_{3} \mathrm{C} \\
\mathrm{H}_{3} \mathrm{C}\end{array}$ & $\Sigma^{-N O}$ \\
\hline Entry & M-catalyst & Solvent & Yield (\%) & $e e(\%)^{c}$ \\
\hline 1 & $\mathrm{Ag}_{2} \mathrm{O}$ & toluene & 90 & 92 \\
\hline 2 & $\mathrm{Ag}_{2} \mathrm{O}$ & $\mathrm{Et}_{2} \mathrm{O}$ & 99 & 92 \\
\hline 3 & $\mathrm{Ag}_{2} \mathrm{O}$ & $\mathrm{CHCl}_{3}$ & 99 & 95 \\
\hline 4 & $\mathrm{Ag}_{2} \mathrm{O}$ & DCE & 99 & 94 \\
\hline 5 & $\mathrm{Ag}_{2} \mathrm{O}$ & $\mathrm{CH}_{2} \mathrm{Cl}_{2}$ & 99 & 95 \\
\hline 6 & $\mathrm{PtCl}_{2}$ & $\mathrm{CH}_{2} \mathrm{Cl}_{2}$ & traces & - \\
\hline 7 & $\mathrm{CuCl}$ & $\mathrm{CH}_{2} \mathrm{Cl}_{2}$ & 37 & 94 \\
\hline 8 & AgOTf & $\mathrm{CH}_{2} \mathrm{Cl}_{2}$ & 25 & 22 \\
\hline 9 & $\mathrm{AgBF}_{4}$ & $\mathrm{CH}_{2} \mathrm{Cl}_{2}$ & 20 & 47 \\
\hline 10 & $\mathrm{Ag}_{2} \mathrm{CO}_{3}$ & $\mathrm{CH}_{2} \mathrm{Cl}_{2}$ & 99 & 95 \\
\hline 11 & $\mathrm{AuClPPh}_{3}$ & $\mathrm{CH}_{2} \mathrm{Cl}_{2}$ & n.d. & - \\
\hline $12^{d}$ & $\mathrm{Ag}_{2} \mathrm{O}$ & $\mathrm{CH}_{2} \mathrm{Cl}_{2}$ & 99 & 96 \\
\hline $13^{e}$ & $\mathrm{Ag}_{2} \mathrm{O}$ & $\mathrm{CH}_{2} \mathrm{Cl}_{2}$ & 99 & 95 \\
\hline $14^{\mathrm{f}}$ & $\mathrm{Ag}_{2} \mathrm{O}$ & $\mathrm{CH}_{2} \mathrm{Cl}_{2}$ & 99 & 96 \\
\hline $15^{g}$ & $\mathrm{Ag}_{2} \mathrm{O}$ & $\mathrm{CH}_{2} \mathrm{Cl}_{2}$ & 99 & 97 \\
\hline
\end{tabular}

${ }^{a}$ Reaction conditions: Dimedone $(\mathbf{1} ; 0.25 \mathrm{mmol})$, nitroalkene $2 \mathrm{2a}(1.1$ equiv), cat. A (1 mol\%), $\mathrm{Ag}_{2} \mathrm{O}(10 \mathrm{~mol} \%)$, solvent ( $\left.2.5 \mathrm{~mL}, 0.1 \mathrm{M}\right)$.

b Yield of 3 a after flash chromatography.

c The enantiomeric excess was determined by HPLC on a chiral stationary phase.

d The reaction was carried out with $\mathbf{A}(0.5 \mathrm{~mol} \%)$.

e The reaction was carried out with $\mathrm{Ag}_{2} \mathrm{O}$ ( 5 mol\%) and $\mathbf{A}$ (0.5 mol\%).

${ }^{f}$ The reaction was carried out with $\mathrm{Ag}_{2} \mathrm{O}$ ( $\left.1 \mathrm{~mol} \%\right)$ and $\mathbf{A}(0.5 \mathrm{~mol} \%)$.

${ }^{9}$ The reaction was carried out with $\mathrm{Ag}_{2} \mathrm{O}(1 \mathrm{~mol} \%)$ and $\mathbf{A}(0.5 \mathrm{~mol} \%)$ at $0{ }^{\circ} \mathrm{C}$.

The substrate scope of the cooperative organo- and silver-catalyzed asymmetric one-pot reaction was then explored for the reaction of dimedone (1) with various alkyne-tethered nitroalkenes $\mathbf{2}$ under optimal reaction conditions (Table 2). The nitroalkenes with electron-withdrawing and electron-donating groups worked smoothly under the cooperative catalysis condition to provide tetrahydrobenzofurans $\mathbf{3 b - f}$ in excellent yields and very good enantioselectivities. The sterically encumbered 1-naphthyl- and 2naphthyl-substituted nitroalkenes led to the formation of the desired tetrahydrobenzofurans $\mathbf{3 g}$,h in very good yields and excellent enantiomeric excesses (Table 2). Furthermore, the one-pot Michael addition/hydroalkoxylation sequence with heteroaryl-substituted nitroalkenes provided the desired annulated product $\mathbf{3 i}$ in excellent yields and enantioselectivity.

Table 2 Substrate Scope

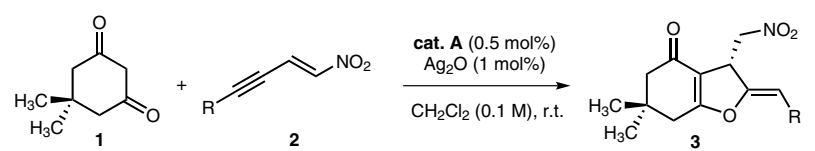

\begin{tabular}{|c|c|c|c|}
\hline 3 & $\mathrm{R}$ & Yield $(\%)^{b}$ & $e e(\%)^{c}$ \\
\hline $\mathbf{a}$ & $\mathrm{Ph}$ & 99 & 96 \\
\hline b & 3- $\mathrm{MeOC}_{6} \mathrm{H}_{4}$ & 98 & 96 \\
\hline c & $2-\mathrm{ClC}_{6} \mathrm{H}_{4}$ & 97 & 95 \\
\hline d & $4-\mathrm{F}_{3} \mathrm{CC}_{6} \mathrm{H}_{4}$ & 93 & 94 \\
\hline e & 3- $\mathrm{MeC}_{6} \mathrm{H}_{4}$ & 97 & 95 \\
\hline f & $3,4-\left(\mathrm{OCH}_{2} \mathrm{O}\right) \mathrm{C}_{6} \mathrm{H}_{3}$ & 97 & 95 \\
\hline $\mathbf{g}$ & 2-naphthyl & 94 & 97 \\
\hline $\mathbf{h}$ & 1-naphthyl & 90 & 95 \\
\hline $\mathbf{i}$ & 2-furanyl & 96 & 96 \\
\hline
\end{tabular}

An extended substrate scope was investigated using different cyclic 1,3-diketones based on five- and six-membered rings. The reaction with 1,3-cyclohexanedione led to the tetrahydrobenzofuran product in good yield and excellent enantioselectivity (Scheme 3, 5a) Interestingly, a dihydropyran derivative $\mathbf{5 b}$ could be obtained in moderate yield and good enantiomeric excess using 1,3-cyclopentanedione. The substrate scope of the cooperative catalytic reaction was extended further to 1,3-diketones bearing heteroatoms, which also provided dihydropyran derivatives in moderate to very good yields and high enantioselectivities (Scheme 3, 5c, d).

The developed one-pot asymmetric transformation was also conducted with various 5-substituted 1,3-cyclohexanediones to introduce another stereocenter via desymmetrization. The desired tetrahydrobenzofurans could be obtained in very good yields and enantioselectivities, but the diastereomeric ratio was virtually $1: 1$ in all attempts (Scheme 4, 7a-d).

To evaluate the efficiency and synthetic utility of the current Michael addition/hydroalkoxylation strategy, tetrahydrobenzofuran 3a was prepared on a gram-scale maintaining the excellent yield and ee value (Scheme 5). 


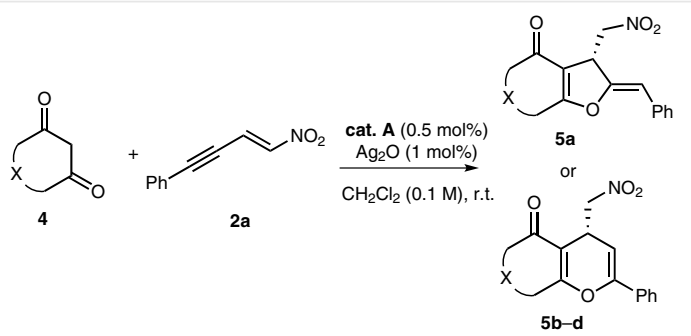<smiles>O=C1CCCC2=C1[C@H](C[N+](=O)[O-])/C(=C/c1ccccc1)O2</smiles><smiles>O=C1CCC2=C1[C@H](C[N+](=O)[O-])C=C(c1ccccc1)O2</smiles><smiles>O=C1COCC2=C1[C@H](C[N+](=O)[O-])C=C(c1ccccc1)O2</smiles><smiles>Cn1c2c(c(=O)n(C)c1=O)[C@@H](C[N+](=O)[O-])C=C(c1ccccc1)O2</smiles>
$\mathbf{5 a}$
$79 \%$ yield $\mathbf{5 b}$
$63 \%$ yield
$94 \%$ ee
$\mathrm{CH}_{3}$

$5 \mathrm{~d}$
$94 \%$ yield

Scheme 3 Extended substrate scope to annulated dihydropyrans

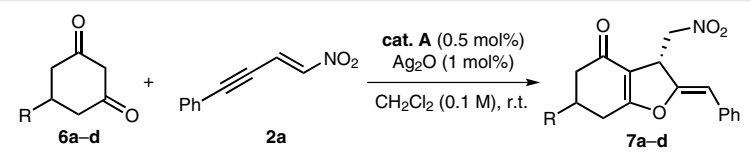<smiles>CC1CC(=O)C2=C(C1)O/C(=C\c1ccccc1)[C@H]2C[N+](=O)[O-]</smiles><smiles>CC(C)C1CC(=O)C2=C(C1)O/C(=C\c1ccccc1)[C@H]2C[N+](=O)[O-]</smiles><smiles>O=C1CC(c2ccccc2)CC2=C1C(C[N+](=O)[O-])C(=Cc1ccccc1)O2</smiles><smiles>O=C1CC(c2ccco2)CC2=C1[C@H](C[N+](=O)[O-])C(=Cc1ccccc1)O2</smiles>

Scheme 4 Extended substrate scope<smiles>CC1(C)[CH+]C(=O)CC(=O)C1</smiles>
$\underset{\mathrm{CH}_{2} \mathrm{Cl}_{2}(0.1 \mathrm{M}) \text {, r.t. }}{\stackrel{\text { cat. }}{A}(0.5 \mathrm{~mol} \%)}$

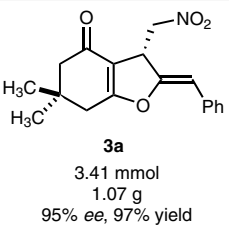

Scheme 5 Gram-scale synthesis of 3a

The absolute configuration of the tetrahydrobenzofurans was determined by X-ray crystal structure analysis of compound 5a (Figure 2 ) $^{18}$ in combination with a CD measurement and calculation (Figure 3 ).

The absolute configuration of the dihydropyran derivatives is based on an X-ray crystallographic analysis of compound $\mathbf{5 d}$ (Figure 4$)^{18}$

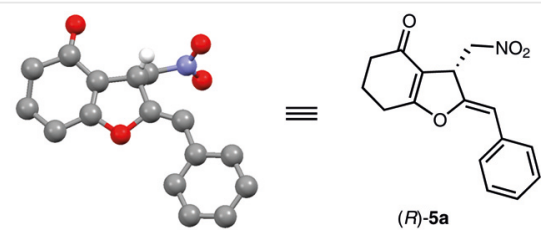

Figure 2 X-ray crystal structure of tetrahydrobenzofuran 5a

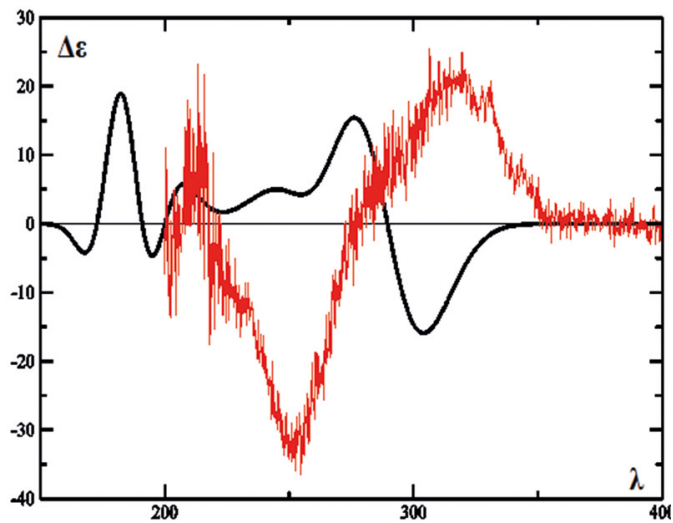

Figure 3 Measured (red) CD spectrum of $\mathbf{5 a}$. The black curve is the calculated CD spectrum of $\mathbf{5} \mathbf{a}$ with the $S$-configuration at carbon atom C3. $\Delta \varepsilon$ in $1000 \mathrm{~cm}^{2} \mathrm{~mol}^{-1}$ and $\lambda$ in nm. For details, see the Supporting Information.

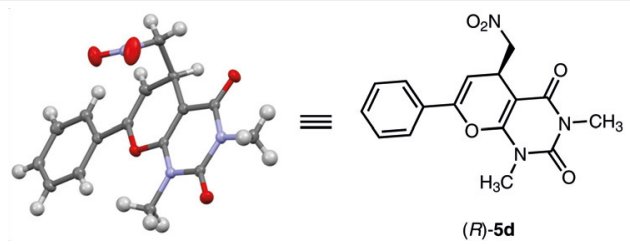

Figure 4 X-ray crystal structure of dihydropyran derivative $\mathbf{5 d}$

This one-pot Michael addition/hydroalkoxylation protocol is proposed to proceed via two catalytic cycles (Scheme 6 ). The first organocatalytic cycle involves the synergistic activation of the 1,3-diketone $\mathbf{1}$ and the nitroalkene $\mathbf{2}$ by the bifunctional squaramide $\mathbf{A}$, where the squaramide moiety activates the nitroalkene $\mathbf{2}$ through the formation of hydrogen bonds to the nitro group and simultaneously the 1,3-diketone undergoes activation by the tertiary amine to promote the Michael addition from the Re-face. In the second catalytic cycle the silver forms a $\pi$-complex for the electrophilic activation of the internal alkyne to facilitate a 5-exo-dig or a 6-endo-dig annulation reaction leading to the vinylsilver intermediate. The latter undergoes a fast protodeargentation to provide the desired product $\mathbf{3 , 5}$ and $\mathbf{7 .}$

In conclusion, we have developed a one-pot asymmetric Michael addition/hydroalkoxylation protocol by merging a bifunctional squaramide and a silver(I) salt at a very low 


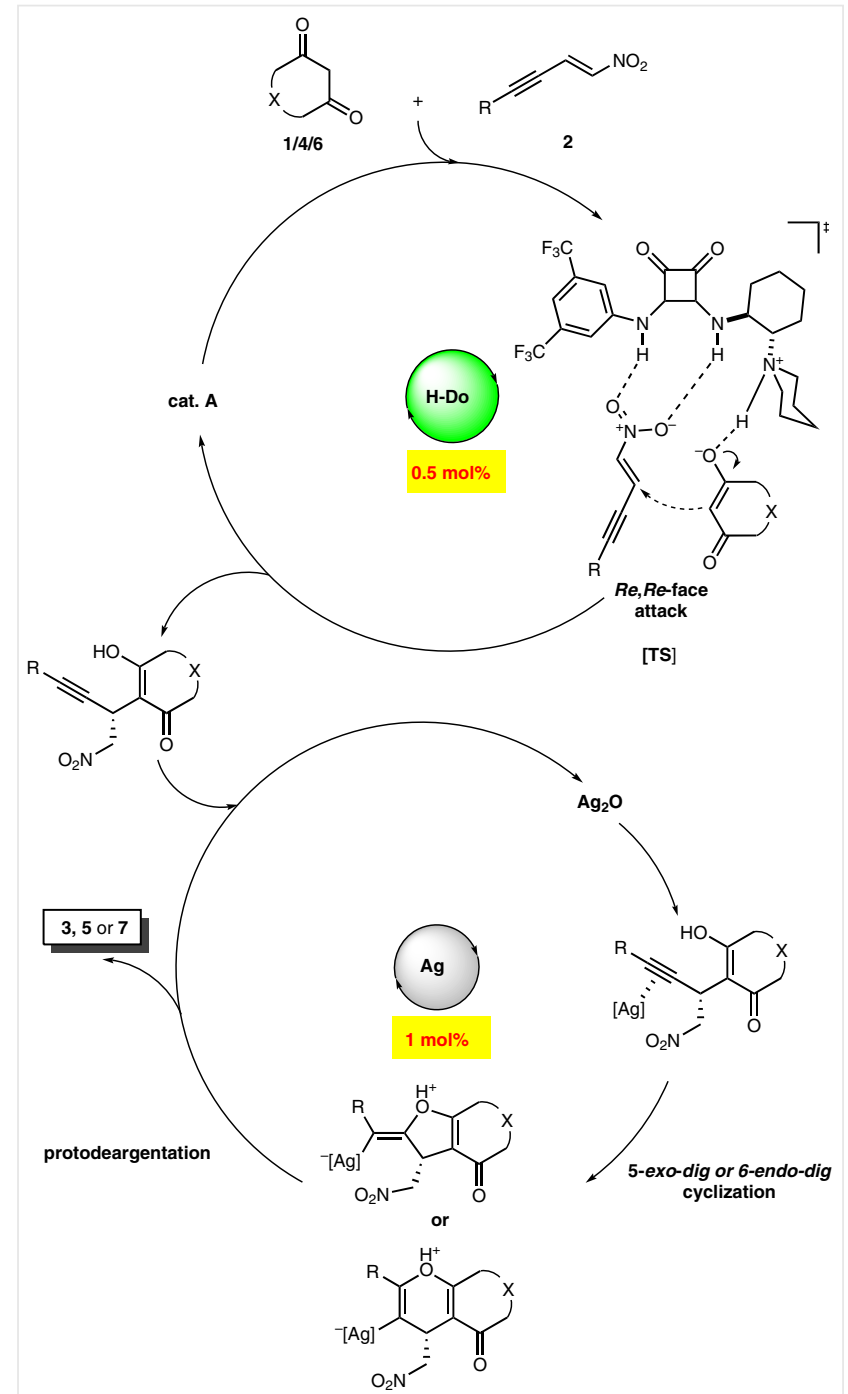

Scheme 6 Proposed mechanism of the one-pot Michael addition/ hydroalkoxylation reaction

catalyst loading. The combination of both catalytic systems enabled the formation of the desired tetrahydrobenzofurans and annulated dihydropyranes in moderate to excellent yields and good to excellent enantiomeric excesses.

Unless otherwise noted, all commercially available chemicals were used without purification. All solvents were distilled and purified according to standard procedures. Analytical TLC was performed using SIL G-25 UV 252 from Macherey \& Nagel (particle size 0.040-0.063 nm; 230-240 mesh. flash) and visualized with ultraviolet radiation at 254 $\mathrm{nm} .{ }^{1} \mathrm{H},{ }^{13} \mathrm{C}$, and ${ }^{19} \mathrm{~F}$ NMR spectra were recorded at ambient temperature on a Varian Innova 400 or Innova 600 spectrometer. Chemical shifts for ${ }^{1} \mathrm{H}$ NMR and ${ }^{13} \mathrm{C}$ NMR spectra are reported in parts per million (ppm) and coupling constants in hertz $(\mathrm{Hz})$. Standard abbreviations are used for the spin multiplicity (qi = quintet). Optical rotations were measured on a PerkinElmer 241 polarimeter. Melting points were measured on a LLG MPM-H2 melting point instrument. Mass spectra were acquired on a Finnigan SSQ7000 (EI, $70 \mathrm{eV}$ ) spectrometer and on a ThermoFinnigan LCQ Deca XP plus (ESI) spectrometer and high-resolution ESI spectra on a ThermoFisher Scientific LTQ Orbitrap XL. Analytical HPLC was performed on a Aligent 1100, Aligent 1260, or Hewlett-Packard 1100 Series instrument using chiral stationary phases (Daicel Chiralpak IC, Daicel Chiralpak IA, Daicel Chiralpak AD, Daicel Chiralpak AS, Daicel Chiralpak IB columns). Analytical SFC was performed on a THAR-SFC MethodStation II with a WATERS 2998 Photodiode Array Detector using chiral stationary phases (Daicel Chiralcel OJ-H). Catalyst $\mathbf{A}$ and $\mathbf{B},{ }^{19}{ }^{\mathbf{D}-\mathbf{I}^{20}}$ and the nitroalkenes $\mathbf{2}^{16 \mathrm{c}}$ were prepared according to known procedures.

\section{Tetrahydrobenzofurans and Annulated Dihydropyrans; General Procedure}

A mixture of 1,3 -diketones $\mathbf{1 , 4}$, or $\mathbf{6}(0.25 \mathrm{mmol})$, nitroalkene $\mathbf{2}(0.275$ mmol, 1.1 equiv), catalyst $\mathbf{A}(0.5 \mathrm{~mol} \%)$, and $\mathrm{Ag}_{2} \mathrm{O}$ (1 mol\%) in $\mathrm{CH}_{2} \mathrm{Cl}_{2}$ $(2.5 \mathrm{~mL}, 0.1 \mathrm{M})$ was stirred at r.t. until the intermediate Michael adduct was completely converted as indicated by TLC. The crude product was directly subjected to flash chromatography on silica (n-pentane $/ \mathrm{Et}_{2} \mathrm{O}$ or $n$-pentane $/ \mathrm{CH}_{2} \mathrm{Cl}_{2}$ ) to afford the corresponding product 3,5 , or 7 .

\section{(R)-(Z)-2-Benzylidene-6,6-dimethyl-3-(nitromethyl)-3,5,6,7-tet- rahydrobenzofuran-4(2H)-one (3a)}

Compound 3a was isolated after flash chromatography (n-pentane/Et $\left.{ }_{2} \mathrm{O}, 1: 1\right)$; yield: $75 \mathrm{mg}(96 \%)$; colorless solid; mp $136-138^{\circ} \mathrm{C}$; $R_{f}=0.22$ (n-pentane/Et $\left.{ }_{2} \mathrm{O}, 1: 1\right) ;[\alpha]_{D}{ }^{24}+96.6(c=0.6, \mathrm{MeOH})$.

HPLC: Daicel Chiralpak IC, $n$-heptane/ $i$-PrOH (7:3), $1.0 \mathrm{~mL} / \mathrm{min}, \lambda=$ $254 \mathrm{~nm}, t_{\mathrm{R}}$ (minor) $=7.6 \mathrm{~min}, t_{\mathrm{R}}$ (major) $=6.4 \mathrm{~min} ; 96 \%$ ee.

IR (ATR): 2955, 2330, 2086, 1900, 1645, 1546, 1492, 1397, 1335, 1286, $1219,1174,1140,1092,998,917,849,755,694 \mathrm{~cm}^{-1}$.

${ }^{1} \mathrm{H}$ NMR $\left(600 \mathrm{MHz}, \mathrm{C}_{6} \mathrm{D}_{6}\right): \delta=7.52(\mathrm{~d}, J=7.4 \mathrm{~Hz}, 2 \mathrm{H}, \mathrm{ArH}), 7.19(\mathrm{t}, J=$ $7.8 \mathrm{~Hz}, 2 \mathrm{H}, \mathrm{ArH}), 7.04(\mathrm{t}, J=7.4 \mathrm{~Hz}, 1 \mathrm{H}, \mathrm{ArH}), 5.42(\mathrm{~d}, J=2.0 \mathrm{~Hz}, 1 \mathrm{H}$, $\mathrm{OC}=\mathrm{CH}), 4.36\left(\mathrm{dd}, J=13.1,6.3 \mathrm{~Hz}, 1 \mathrm{H}, \mathrm{CHHNO}_{2}\right), 4.17(\mathrm{dd}, J=13.1,4.7$ $\left.\mathrm{Hz}, 1 \mathrm{H}, \mathrm{CHHNO}_{2}\right), 3.92\left(\mathrm{~s}, 1 \mathrm{H}, \mathrm{CHCH}_{2}\right), 1.93\left(\mathrm{~d}, J=16.0 \mathrm{~Hz}, 1 \mathrm{H}, \mathrm{CH}_{2}\right)$, $1.85\left(\mathrm{~d}, J=16.0 \mathrm{~Hz}, 1 \mathrm{H}, \mathrm{CH}_{2}\right), 1.66\left(\mathrm{~d}, J=17.9 \mathrm{~Hz}, 1 \mathrm{H}, \mathrm{CH}_{2}\right), 1.58(\mathrm{~d}, J=$ $\left.17.9 \mathrm{~Hz}, 1 \mathrm{H}, \mathrm{CH}_{2}\right), 0.64\left(\mathrm{~s}, 3 \mathrm{H}, \mathrm{CH}_{3}\right), 0.58\left(\mathrm{~s}, 3 \mathrm{H}, \mathrm{CH}_{3}\right)$.

${ }^{13} \mathrm{C}$ NMR (151 MHz, $\left.\mathrm{C}_{6} \mathrm{D}_{6}\right): \delta=191.6(\mathrm{C}=\mathrm{O}), 173.4\left(\mathrm{C}_{\mathrm{q}}\right), 153.4\left(\mathrm{C}_{\mathrm{q}}\right)$, $133.9\left(\mathrm{C}_{\mathrm{q}}\right), 128.7$ (2 C, $\left.\operatorname{ArC}\right), 128.3(2 \mathrm{C}, \operatorname{ArC}), 127.0(\operatorname{ArC}), 111.1\left(\mathrm{C}_{\mathrm{q}}\right)$, 106.0 $(\mathrm{OC}=\mathrm{CH}), 75.3\left(\mathrm{CH}_{2} \mathrm{NO}_{2}\right), 50.5\left(\mathrm{CH}_{2}\right), 41.9\left(\mathrm{CHCH}_{2}\right), 36.1\left(\mathrm{CH}_{2}\right)$, $33.2\left[\mathrm{C}\left(\mathrm{CH}_{3}\right)_{2}\right], 28.4\left(\mathrm{CH}_{3}\right), 27.2\left(\mathrm{CH}_{3}\right)$.

MS (EI, $70 \mathrm{eV}): m / z(\%)=313.1\left(1,[\mathrm{M}]^{+}\right), 267.1\left(17,\left[\mathrm{M}-\mathrm{NO}_{2}\right]^{+}\right), 253.0$ (7, $\left.\left[\mathrm{M}-\mathrm{CH}_{2} \mathrm{NO}_{2}\right]^{+}\right), 90.1\left(38,\left[\mathrm{CH}_{2} \mathrm{C}_{6} \mathrm{H}_{5}\right]^{+}\right), 77.1\left(27,\left[\mathrm{C}_{6} \mathrm{H}_{5}\right]^{+}\right)$.

HRMS $\left(\mathrm{ESI}^{+}\right): \mathrm{m} / z[\mathrm{M}+\mathrm{Na}]^{+}$calcd for $\mathrm{C}_{18} \mathrm{H}_{19} \mathrm{NO}_{4} \mathrm{Na}$ : 336.1206; found: 336.1195.

(R)-(Z)-2-(3-Methoxybenzylidene)-6,6-dimethyl-3-(nitromethyl)3,5,6,7-tetrahydrobenzofuran-4(2H)-one (3b)

Compound $\mathbf{3 b}$ was isolated after flash chromatography (n-pentane/Et ${ }_{2} \mathrm{O}, 2: 1$ ); yield: $84 \mathrm{mg}(98 \%)$; colorless solid; mp $127-129^{\circ} \mathrm{C}$; $R_{f}=0.17$ ( $n$-pentane/Et $\left.{ }_{2} \mathrm{O}, 1: 1\right) ;[\alpha]_{D}{ }^{24}+67.7(c=0.4$, benzene) .

HPLC: Daicel Chiralpak IC, $n$-heptane $/ i$-PrOH (7:3), $0.7 \mathrm{~mL} / \mathrm{min}, \lambda=$ $254 \mathrm{~nm}, t_{\mathrm{R}}$ (minor) $=14.0 \mathrm{~min}, t_{\mathrm{R}}$ (major) $=15.4 \mathrm{~min}$; $96 \%$ ee.

IR (ATR): 2934, 2293, 2090, 1891, 1649, 1566, 1399, 1232, 1015, 840, $692 \mathrm{~cm}^{-1}$. 
${ }^{1} \mathrm{H}$ NMR $\left(600 \mathrm{MHz}, \mathrm{CDCl}_{3}\right): \delta=7.25(\mathrm{~m}, 1 \mathrm{H}, \mathrm{ArH}), 7.11(\mathrm{~m}, 2 \mathrm{H}, \mathrm{ArH})$, 6.83-6.78 (m, $1 \mathrm{H}, \mathrm{ArH}), 5.71(\mathrm{~d}, J=2.2 \mathrm{~Hz}, 1 \mathrm{H}, \mathrm{OC}=\mathrm{CH}), 4.89$ (dd, $J=$ 13.3, $3.9 \mathrm{~Hz}, 1 \mathrm{H}, \mathrm{CHHNO}_{2}$ ), 4.73 (dd, $J=13.3,7.1 \mathrm{~Hz}, 1 \mathrm{H}, \mathrm{CHHNO}_{2}$ ), 4.58-4.53 (m, $\left.1 \mathrm{H}, \mathrm{CHCH}_{2}\right), 3.82\left(\mathrm{~s}, 3 \mathrm{H}, \mathrm{OCH}_{3}\right), 2.54\left(\mathrm{~m}, 2 \mathrm{H}, \mathrm{CH}_{2}\right), 2.31$ ( $\left.\mathrm{m}, 2 \mathrm{H}, \mathrm{CH}_{2}\right), 1.17$ (s, $\left.3 \mathrm{H}, \mathrm{CH}_{3}\right), 1.16\left(\mathrm{~s}, 3 \mathrm{H}, \mathrm{CH}_{3}\right)$.

${ }^{13} \mathrm{C}$ NMR (151 MHz, $\left.\mathrm{CDCl}_{3}\right): \delta=193.6(\mathrm{C}=0), 175.0\left(\mathrm{C}_{\mathrm{q}}\right), 159.6\left(\mathrm{C}_{\mathrm{q}}\right)$, $153.3\left(C_{q}\right), 134.5\left(C_{q}\right), 129.4(\operatorname{ArC}), 121.3(\operatorname{ArC}), 114.2(\operatorname{ArC}), 112.8$ (ArC), $111.3\left(\mathrm{C}_{\mathrm{q}}\right), 106.7(\mathrm{OC}=\mathrm{CH}), 75.7\left(\mathrm{CH}_{2} \mathrm{NO}_{2}\right), 55.2\left(\mathrm{OCH}_{3}\right), 51.0$ $\left(\mathrm{CH}_{2}\right), 41.7\left(\mathrm{CHCH}_{2}\right), 37.2\left(\mathrm{CH}_{2}\right), 34.4\left[\mathrm{C}\left(\mathrm{CH}_{3}\right)_{2}\right], 29.0\left(\mathrm{CH}_{3}\right), 28.3\left(\mathrm{CH}_{3}\right)$. MS (EI, $70 \mathrm{eV}): m / z(\%)=343.3\left(5,[\mathrm{M}]^{+}\right), 297.3\left(34,\left[\mathrm{M}-\mathrm{NO}_{2}\right]^{+}\right), 283.3$ $\left(12,\left[\mathrm{M}-\mathrm{CH}_{2} \mathrm{NO}_{2}\right]^{+}\right)$.

HRMS (ESI $\left.{ }^{+}\right): m / z[\mathrm{M}+\mathrm{Na}]^{+}$calcd for $\mathrm{C}_{19} \mathrm{H}_{21} \mathrm{NO}_{5} \mathrm{Na}$ : 366.1312 ; found: 366.1310 .

\section{(R)-(Z)-2-(2-Chlorobenzylidene)-6,6-dimethyl-3-(nitromethyl)- 3,5,6,7-tetrahydrobenzofuran- $4(2 \mathrm{H})$-one $(3 \mathrm{c})$}

Compound $\mathbf{3 c}$ was isolated after flash chromatography (n-pentane/Et $\left.{ }_{2} \mathrm{O}, 1: 1\right)$; yield: $84 \mathrm{mg}(97 \%)$; colorless solid; mp $130-132{ }^{\circ} \mathrm{C}$; $R_{f}=0.36$ (n-pentane/ $\left.\mathrm{Et}_{2} \mathrm{O}, 1: 1\right) ;[\alpha]_{\mathrm{D}}{ }^{24}=115.8$ ( $c=0.5$, benzene).

HPLC: Daicel Chiralpak IA, $n$-heptane/i-PrOH (7:3), $0.7 \mathrm{~mL} / \mathrm{min}, \lambda=$ $230 \mathrm{~nm}, t_{\mathrm{R}}($ minor $)=8.4 \mathrm{~min}, t_{\mathrm{R}}$ (major) $=9.0 \mathrm{~min} ; 95 \%$ ee.

IR (ATR): 2956, 2314, 2084, 1648, 1552, 1396, 1284, 1214, 1017, 972 , $852,754,692 \mathrm{~cm}^{-1}$.

${ }^{1} \mathrm{H}$ NMR $\left(600 \mathrm{MHz}, \mathrm{C}_{6} \mathrm{D}_{6}\right): \delta=7.95$ (dd, $\left.J=7.9,1.6 \mathrm{~Hz}, 1 \mathrm{H}, \mathrm{ArH}\right), 7.17$ (dd, $J=8.1,1.2 \mathrm{~Hz}, 1 \mathrm{H}, \mathrm{ArH}), 6.97(\mathrm{~m}, 1 \mathrm{H}, \mathrm{ArH}), 6.73(\mathrm{~m}, 1 \mathrm{H}, \mathrm{ArH})$, $6.04(\mathrm{~d}, J=2.3 \mathrm{~Hz}, 1 \mathrm{H}, \mathrm{OC}=\mathrm{CH}), 4.48(\mathrm{dd}, J=13.2,5.3 \mathrm{~Hz}, 1 \mathrm{H}, \mathrm{CHH}-$ $\mathrm{NO}_{2}$ ), 3.99 (dd, $J=13.2,3.6 \mathrm{~Hz}, 1 \mathrm{H}, \mathrm{CHHNO}$ ), $3.69\left(\mathrm{~s}, 1 \mathrm{H}, \mathrm{CHCH}_{2}\right.$ ), $1.92\left(\mathrm{~d}, J=16.0 \mathrm{~Hz}, 1 \mathrm{H}, \mathrm{CH}_{2}\right), 1.83\left(\mathrm{~d}, J=16.0 \mathrm{~Hz}, 1 \mathrm{H}, \mathrm{CH}_{2}\right), 1.67(\mathrm{~d}, J=$ $\left.17.9 \mathrm{~Hz}, 1 \mathrm{H}, \mathrm{CH}_{2}\right), 1.57$ (d, J = $\left.17.9 \mathrm{~Hz}, 1 \mathrm{H}, \mathrm{CH}_{2}\right), 0.64\left(\mathrm{~s}, 3 \mathrm{H}, \mathrm{CH}_{3}\right)$, $0.56\left(\mathrm{~s}, 3 \mathrm{H}, \mathrm{CH}_{3}\right)$.

${ }^{13} \mathrm{C}$ NMR (151 MHz, $\left.\mathrm{C}_{6} \mathrm{D}_{6}\right): \delta=191.6(\mathrm{C}=\mathrm{O}), 173.2\left(\mathrm{C}_{\mathrm{q}}\right), 155.4\left(\mathrm{C}_{\mathrm{q}}\right)$, $132.7\left(\mathrm{C}_{\mathrm{q}}\right), 131.9\left(\mathrm{C}_{\mathrm{q}}\right), 130.2(\operatorname{ArC}), 129.4(\operatorname{ArC}), 128.1(\operatorname{ArC}), 126.5$ (ArC), $111.4\left(\mathrm{C}_{\mathrm{q}}\right), 101.5(\mathrm{OC}=\mathrm{CH}), 75.2\left(\mathrm{CH}_{2} \mathrm{NO}_{2}\right), 50.5\left(\mathrm{CH}_{2}\right), 42.0$ $\left(\mathrm{CHCH}_{2}\right), 36.1\left(\mathrm{CH}_{2}\right), 33.2\left[\mathrm{C}\left(\mathrm{CH}_{3}\right)_{2}\right], 28.4\left(\mathrm{CH}_{3}\right), 27.2\left(\mathrm{CH}_{3}\right)$.

MS (EI, $70 \mathrm{eV}): m / z(\%)=348.3\left(1,[\mathrm{M}+\mathrm{H}]^{+}\right), 303.3\left(7,\left[\mathrm{M}-\mathrm{NO}_{2},{ }^{37} \mathrm{Cl}\right]^{+}\right)$, $301.2\left(37,\left[\mathrm{M}-\mathrm{NO}_{2},{ }^{35} \mathrm{Cl}\right]^{+}\right), 289.2\left(2,\left[\mathrm{M}-\mathrm{CH}_{2} \mathrm{NO}_{2},{ }^{37} \mathrm{Cl}\right]^{+}\right), 287.2(6,[\mathrm{M}$ $\left.\left.-\mathrm{NO}_{2},{ }^{35} \mathrm{Cl}\right]^{+}\right)$.

HRMS $\left(\mathrm{ESI}^{+}\right): m / z[\mathrm{M}+\mathrm{Na}]^{+}$calcd for $\mathrm{C}_{18} \mathrm{H}_{18} \mathrm{ClNO}_{4} \mathrm{Na}$ : 370.0812; found: 370.0813 .

(R)-(Z)-6,6-Dimethyl-3-(nitromethyl)-2-[4-(trifluoromethyl)benzylidene]-3,5,6,7-tetrahydrobenzofuran- $4(2 \mathrm{H})$-one (3d)

Compound 3d was isolated after flash chromatography (n-pentane/ $\left.\mathrm{Et}_{2} \mathrm{O}, 1: 2\right)$; yield: $106 \mathrm{mg}(93 \%)$; colorless solid; $\mathrm{mp} 54-56{ }^{\circ} \mathrm{C}$; $R_{f}=0.14$ ( $n$-pentane $\left./ \mathrm{Et}_{2} \mathrm{O}, 1: 1\right) ;[\alpha]_{D}{ }^{24}+49.2$ ( $c=0.3$, benzene).

HPLC: Daicel Chiralpak IC, $n$-heptane $/ i-P r O H ~(8: 2), 1.0 \mathrm{~mL} / \mathrm{min}, \lambda=$ $254 \mathrm{~nm}, t_{\mathrm{R}}($ minor $)=7.9 \mathrm{~min}, t_{\mathrm{R}}$ (major) $=6.1 \mathrm{~min} ; 94 \%$ ee.

IR (ATR): 2962, 1949, 1692, 1651, 1615, 1552, 1400, 1321, 1219, 1166, 1116, 1067, 1014, 917, 862, 834, 791, 758, $703 \mathrm{~cm}^{-1}$.

${ }^{1} \mathrm{H}$ NMR $\left(400 \mathrm{MHz}, \mathrm{CDCl}_{3}\right): \delta=7.59(\mathrm{~m}, 4 \mathrm{H}, \mathrm{ArH}), 5.76(\mathrm{~d}, J=2.2 \mathrm{~Hz}, 1$ $\mathrm{H}, \mathrm{OC}=\mathrm{CH}), 4.90\left(\mathrm{dd}, J=13.4,3.9 \mathrm{~Hz}, 1 \mathrm{H}, \mathrm{CHHNO}_{2}\right), 4.75$ (dd, $J=13.4$, $\left.6.9 \mathrm{~Hz}, 1 \mathrm{H}, \mathrm{CHHNO}_{2}\right), 4.55\left(\mathrm{~m}, 1 \mathrm{H}, \mathrm{CHCH}_{2}\right), 2.55\left(\mathrm{~m}, 2 \mathrm{H}, \mathrm{CH}_{2}\right), 2.31(\mathrm{~s}$, $\left.2 \mathrm{H}, \mathrm{CH}_{2}\right), 1.17\left(\mathrm{~s}, 3 \mathrm{H}, \mathrm{CH}_{3}\right), 1.16\left(\mathrm{~s}, 3 \mathrm{H}, \mathrm{CH}_{3}\right)$.

${ }^{13} \mathrm{C}$ NMR $\left(101 \mathrm{MHz}, \mathrm{CDCl}_{3}\right): \delta=193.5(\mathrm{C}=\mathrm{O}), 174.7\left(\mathrm{C}_{\mathrm{q}}\right), 155.0\left(\mathrm{C}_{\mathrm{q}}\right)$, $136.8\left(\mathrm{C}_{\mathrm{q}}\right), 128.7$ (3 C, $\left.\operatorname{ArC}\right), 125.3(2 \mathrm{C}, \operatorname{ArC}), 122.7\left(\mathrm{CF}_{3}\right), 111.4\left(\mathrm{C}_{\mathrm{q}}\right)$, $105.4(\mathrm{OC}=\mathrm{CH}), 75.4\left(\mathrm{CH}_{2} \mathrm{NO}_{2}\right), 51.0\left(\mathrm{CH}_{2}\right), 41.9\left(\mathrm{CHCH}_{2}\right), 37.2\left(\mathrm{CH}_{2}\right)$, $34.4\left[\mathrm{C}\left(\mathrm{CH}_{3}\right)_{2}\right], 28.9\left(\mathrm{CH}_{3}\right), 28.2\left(\mathrm{CH}_{3}\right)$.
${ }^{19} \mathrm{~F}$ NMR (376 MHz, $\left.\mathrm{CDCl}_{3}\right): \delta=-62.61(\mathrm{~s})$.

MS (EI, $70 \mathrm{eV}): m / z(\%)=382.3\left(3,[\mathrm{M}+\mathrm{H}]^{+}\right), 335.3\left(29,\left[\mathrm{M}-\mathrm{NO}_{2}\right]^{+}\right)$, $321.2\left(9,\left[\mathrm{M}-\mathrm{CH}_{2} \mathrm{NO}_{2}\right]^{+}\right)$.

HRMS $\left(\mathrm{ESI}^{+}\right): m / z[\mathrm{M}+\mathrm{Na}]^{+}$calcd for $\mathrm{C}_{19} \mathrm{H}_{18} \mathrm{~F}_{3} \mathrm{NO}_{4} \mathrm{Na}$ : 404.1080; found: 404.1069 .

(R)-(Z)-6,6-Dimethyl-2-(3-methylbenzylidene)-3-(nitromethyl)3,5,6,7-tetrahydrobenzofuran-4(2H)-one (3e)

Compound 3e was isolated after flash chromatography (n-pentane/Et $\left.{ }_{2} \mathrm{O}, 1: 1\right)$; yield: $79 \mathrm{mg}(97 \%)$; colorless solid; mp $138-140{ }^{\circ} \mathrm{C}$; $R_{f}=0.26$ ( $n$-pentane/Et $\left.{ }_{2} \mathrm{O}, 1: 1\right) ;[\alpha]_{D}{ }^{24}+71.2(c=0.4$, benzene).

HPLC: Daicel Chiralpak IB, $n$-heptane $/ i$-PrOH (7:3), $0.7 \mathrm{~mL} / \mathrm{min}, \lambda=$ $254 \mathrm{~nm}, t_{\mathrm{R}}($ minor $)=11.0 \mathrm{~min}, t_{\mathrm{R}}$ (major $)=10.0 \mathrm{~min} ; 95 \%$ ee .

IR (ATR): 2957, 2290, 2086, 1644, 1547, 1474, 1403, 1328, 1280, 1214, $1171,1093,1006,891,840,781,697 \mathrm{~cm}^{-1}$.

${ }^{1} \mathrm{H}$ NMR $\left(600 \mathrm{MHz}, \mathrm{CDCl}_{3}\right): \delta=7.37(\mathrm{~d}, J=7.8 \mathrm{~Hz}, 1 \mathrm{H}, \mathrm{ArH}), 7.32(\mathrm{~s}, 1$ $\mathrm{H}, \mathrm{ArH}), 7.23(\mathrm{t}, J=7.7 \mathrm{~Hz}, 1 \mathrm{H}, \mathrm{ArH}), 7.06(\mathrm{~d}, J=7.5 \mathrm{~Hz}, 1 \mathrm{H}, \mathrm{ArH}), 5.70$ $(\mathrm{d}, J=2.2 \mathrm{~Hz}, 1 \mathrm{H}, \mathrm{OC}=\mathrm{CH}), 4.89$ (dd, $J=13.2,3.9 \mathrm{~Hz}, 1 \mathrm{H}, \mathrm{CHHNO}_{2}$ ), $4.73\left(\mathrm{dd}, J=13.3,7.1 \mathrm{~Hz}, 1 \mathrm{H}, \mathrm{CHHNO}_{2}\right), 4.55\left(\mathrm{~m}, 1 \mathrm{H}, \mathrm{CHCH}_{2}\right), 2.55(\mathrm{~m}$, $\left.2 \mathrm{H}, \mathrm{CH}_{2}\right), 2.35\left(\mathrm{~s}, 3 \mathrm{H}, \mathrm{CH}_{3}\right), 2.32\left(\mathrm{~m}, 2 \mathrm{H}, \mathrm{CH}_{2}\right), 1.17\left(\mathrm{~s}, 3 \mathrm{H}, \mathrm{CH}_{3}\right), 1.16$ (s, $3 \mathrm{H}, \mathrm{CH}_{3}$ ).

${ }^{13} \mathrm{C}$ NMR $\left(151 \mathrm{MHz}, \mathrm{CDCl}_{3}\right): \delta=193.6(\mathrm{C}=0), 175.1\left(\mathrm{C}_{\mathrm{q}}\right), 152.8\left(\mathrm{C}_{\mathrm{q}}\right)$, $138.0\left(C_{q}\right), 133.2\left(C_{q}\right), 129.4(\operatorname{ArC}), 128.4(\operatorname{ArC}), 128.2(\operatorname{ArC}), 125.7$ $(\operatorname{ArC}), 111.3\left(\mathrm{C}_{\mathrm{q}}\right), 106.9(\mathrm{OC}=\mathrm{CH}), 75.7\left(\mathrm{CH}_{2} \mathrm{NO}_{2}\right), 51.0\left(\mathrm{CH}_{2}\right), 41.7$ $\left(\mathrm{CHCH}_{2}\right), 37.3\left(\mathrm{CH}_{2}\right), 34.4\left[\mathrm{C}\left(\mathrm{CH}_{3}\right)_{2}\right], 29.0\left(\mathrm{CH}_{3}\right), 28.3\left(\mathrm{CH}_{3}\right), 21.5$ $\left(\mathrm{ArCH}_{3}\right)$.

MS (EI, $70 \mathrm{eV}): m / z(\%)=327.2\left(2,[\mathrm{M}]^{+}\right), 328.2\left(9,[\mathrm{M}+\mathrm{H}]^{+}\right), 281.3(26$, $\left.\left[\mathrm{M}-\mathrm{NO}_{2}\right]^{+}\right), 267.3\left(17,\left[\mathrm{M}-\mathrm{CH}_{2} \mathrm{NO}_{2}\right]^{+}\right)$.

HRMS (ESI $\left.{ }^{+}\right): m / z[\mathrm{M}+\mathrm{Na}]^{+}$calcd for $\mathrm{C}_{19} \mathrm{H}_{21} \mathrm{NO}_{4} \mathrm{Na}$ : 350.1363 ; found: 350.1356

(R)-(Z)-2-(Benzo[d][1,3]dioxol-5-ylmethylene)-6,6-dimethyl-3(nitromethyl)-3,5,6,7-tetrahydrobenzofuran-4(2H)-one (3f)

Compound 3f was isolated after flash chromatography ( $n$-pentane/Et $\left.{ }_{2} \mathrm{O}, 1: 2\right)$; yield: $87 \mathrm{mg}(97 \%)$; colorless solid; $\mathrm{mp} 138-140{ }^{\circ} \mathrm{C}$; $R_{f}=0.21$ ( $n$-pentane $\left./ \mathrm{Et}_{2} \mathrm{O}, 1: 1\right) ;[\alpha]_{D}{ }^{24}+72.8(c=0.4$, benzene).

HPLC: Daicel Chiralpak IB, $n$-heptane/EtOH (7:3), $1.0 \mathrm{~mL} / \mathrm{min}, \lambda=254$ $\mathrm{nm}, t_{\mathrm{R}}$ (minor) $=9.8 \mathrm{~min}, t_{\mathrm{R}}$ (major) $=13.8 \mathrm{~min} ; 95 \%$ ee.

IR (ATR): 2960, 1650, 1549, 1493, 1398, 1292, 1239, 1097, 1027, 928 , $875,804,697 \mathrm{~cm}^{-1}$.

${ }^{1} \mathrm{H}$ NMR $\left(600 \mathrm{MHz}, \mathrm{C}_{6} \mathrm{D}_{6}\right): \delta=7.42(\mathrm{~m}, 1 \mathrm{H}, \mathrm{ArH}), 6.72(\mathrm{~m}, 1 \mathrm{H}, \mathrm{ArH})$, $6.64(\mathrm{~m}, 1 \mathrm{H}, \mathrm{ArH}), 5.36(\mathrm{~d}, J=2.1 \mathrm{~Hz}, 1 \mathrm{H}, \mathrm{OC}=\mathrm{CH}), 5.27(\mathrm{~m}, 2 \mathrm{H}$, $\left.\mathrm{OCH}_{2} \mathrm{O}\right), 4.31\left(\mathrm{dd}, J=12.9,6.4 \mathrm{~Hz}, 1 \mathrm{H}, \mathrm{CHHNO}_{2}\right), 4.18(\mathrm{dd}, J=13.0,3.8$ $\left.\mathrm{Hz}, 1 \mathrm{H}, \mathrm{CHHNO}_{2}\right), 3.94\left(\mathrm{~s}, 1 \mathrm{H}, \mathrm{CHCH}_{2}\right), 1.92\left(\mathrm{~d}, J=16.0 \mathrm{~Hz}, 1 \mathrm{H}, \mathrm{CH}_{2}\right)$, $1.84\left(\mathrm{~d}, J=16.0 \mathrm{~Hz}, 1 \mathrm{H}, \mathrm{CH}_{2}\right), 1.56\left(\mathrm{~d}, J=17.9 \mathrm{~Hz}, 1 \mathrm{H}, \mathrm{CH}_{2}\right), 1.48(\mathrm{~d}, J=$ $\left.17.9 \mathrm{~Hz}, 1 \mathrm{H}, \mathrm{CH}_{2}\right), 0.63\left(\mathrm{~s}, 3 \mathrm{H}, \mathrm{CH}_{3}\right), 0.57\left(\mathrm{~s}, 3 \mathrm{H}, \mathrm{CH}_{3}\right)$.

${ }^{13} \mathrm{C}$ NMR (151 MHz, $\left.\mathrm{C}_{6} \mathrm{D}_{6}\right): \delta=191.7(\mathrm{C}=\mathrm{O}), 173.4\left(\mathrm{C}_{\mathrm{q}}\right), 151.9\left(\mathrm{C}_{\mathrm{q}}\right)$, $148.1\left(\mathrm{C}_{\mathrm{q}}\right), 146.8\left(\mathrm{C}_{\mathrm{q}}\right), 128.1\left(\mathrm{C}_{\mathrm{q}}\right), 123.1(\operatorname{ArC}), 111.0\left(\mathrm{C}_{\mathrm{q}}\right), 108.6(\operatorname{ArC})$, 108.2 ( $\mathrm{ArC}), 106.0(\mathrm{OC}=\mathrm{CH}), 100.8\left(\mathrm{OCH}_{2} \mathrm{O}\right), 75.4\left(\mathrm{CH}_{2} \mathrm{NO}_{2}\right), 50.5\left(\mathrm{CH}_{2}\right)$, $41.8\left(\mathrm{CHCH}_{2}\right), 36.0\left(\mathrm{CH}_{2}\right), 33.1\left[\mathrm{C}\left(\mathrm{CH}_{3}\right)_{2}\right], 28.3\left(\mathrm{CH}_{3}\right), 27.2\left(\mathrm{CH}_{3}\right)$.

MS (EI, $70 \mathrm{eV}): m / z(\%)=358.3\left(5,[\mathrm{M}+\mathrm{H}]^{+}\right), 357.3\left(17,[\mathrm{M}]^{+}\right), 311.3$ $\left(24,\left[\mathrm{M}-\mathrm{NO}_{2}\right]^{+}\right), 297.3\left(28,\left[\mathrm{M}-\mathrm{CH}_{2} \mathrm{NO}_{2}\right]^{+}\right)$.

HRMS (ESI $\left.{ }^{+}\right): m / z[\mathrm{M}+\mathrm{Na}]^{+}$calcd for $\mathrm{C}_{19} \mathrm{H}_{19} \mathrm{NO}_{6} \mathrm{Na}$ : 380.1105; found: 380.1094 . 
(R)-(Z)-6,6-Dimethyl-2-(naphthalen-2-ylmethylene)-3-(nitromethyl)-3,5,6,7-tetrahydrobenzofuran-4(2H)-one (3g)

Compound $\mathbf{3 g}$ was isolated after flash chromatography (n-pentane/ $\mathrm{Et}_{2} \mathrm{O}, 1: 1$ to $\left.1: 2\right)$; yield: $85 \mathrm{mg}(94 \%)$; colorless solid; mp $133-$ $135^{\circ} \mathrm{C} ; R_{f}=0.24$ ( $n$-pentane/Et $\left.{ }_{2} \mathrm{O}, 1: 1\right) ;[\alpha]_{\mathrm{D}}{ }^{24}+93.9$ ( $c=0.4$, benzene). HPLC: Daicel Chiralpak IA, $n$-heptane $/ i-P r O H ~(7: 3), 0.7 \mathrm{~mL} / \mathrm{min}, \lambda=$ $254 \mathrm{~nm}, t_{\mathrm{R}}($ minor $)=11.1 \mathrm{~min}, t_{\mathrm{R}}$ (major) $=11.7 \mathrm{~min}$; $97 \%$ ee.

IR (ATR): 2956, 2313, 2073, 2002, 1646, 1549, 1463, 1401, 1290, 1218 , $1173,1140,1090,1019,900,862,821,749,699 \mathrm{~cm}^{-1}$.

${ }^{1} \mathrm{H}$ NMR $\left(600 \mathrm{MHz}, \mathrm{CDCl}_{3}\right): \delta=7.94(\mathrm{~s}, 1 \mathrm{H}, \mathrm{ArH}), 7.84-7.77(\mathrm{~m}, 3 \mathrm{H}$, ArH), 7.72 (dd, J= 8.6, $1.7 \mathrm{~Hz}, 1 \mathrm{H}, \mathrm{ArH}), 7.50-7.43$ (m, $2 \mathrm{H}, \mathrm{ArH}), 5.90$ (d, $J=2.1 \mathrm{~Hz}, 1 \mathrm{H}, \mathrm{OC}=\mathrm{CH}), 4.94\left(\mathrm{dd}, J=13.3,3.9 \mathrm{~Hz}, 1 \mathrm{H}, \mathrm{CHHNO}_{2}\right)$, $4.77\left(\mathrm{dd}, J=13.3,7.2 \mathrm{~Hz}, 1 \mathrm{H}, \mathrm{CHHNO}_{2}\right), 4.62\left(\mathrm{~m}, 1 \mathrm{H}, \mathrm{CHCH}_{2}\right), 2.60(\mathrm{~m}$, $\left.2 \mathrm{H}, \mathrm{CH}_{2}\right), 2.34\left(\mathrm{~s}, 2 \mathrm{H}, \mathrm{CH}_{2}\right), 1.19\left(\mathrm{~s}, 3 \mathrm{H}, \mathrm{CH}_{3}\right), 1.18\left(\mathrm{~s}, 3 \mathrm{H}, \mathrm{CH}_{3}\right)$.

${ }^{13} \mathrm{C}$ NMR (151 MHz, $\left.\mathrm{CDCl}_{3}\right): \delta=193.6(\mathrm{C}=\mathrm{O}), 175.0\left(\mathrm{C}_{\mathrm{q}}\right), 153.3\left(\mathrm{C}_{\mathrm{q}}\right)$, $133.4\left(C_{\mathrm{q}}\right), 132.4\left(\mathrm{C}_{\mathrm{q}}\right), 130.9\left(\mathrm{C}_{\mathrm{q}}\right), 128.1(\operatorname{ArC}), 128.0(\operatorname{ArC}), 127.8(\operatorname{ArC})$, 127.6 (ArC), $126.5(\operatorname{ArC}), 126.3(\operatorname{ArC}), 126.1(\operatorname{ArC}), 111.4\left(C_{\mathrm{q}}\right), 106.9$ $(\mathrm{OC}=\mathrm{CH}), 75.7\left(\mathrm{CH}_{2} \mathrm{NO}_{2}\right), 51.1\left(\mathrm{CH}_{2}\right), 41.8\left(\mathrm{CHCH}_{2}\right), 37.3\left(\mathrm{CH}_{2}\right), 34.4$ $\left[\mathrm{C}\left(\mathrm{CH}_{3}\right)_{2}\right], 29.0\left(\mathrm{CH}_{3}\right), 28.3\left(\mathrm{CH}_{3}\right)$.

MS (EI, $70 \mathrm{eV}): m / z(\%)=364.3\left(2,[\mathrm{M}+\mathrm{H}]^{+}\right), 363.3\left(4,[\mathrm{M}]^{+}\right), 317.3(27$, $\left.\left[\mathrm{M}-\mathrm{NO}_{2}\right]^{+}\right), 303.3\left(12,\left[\mathrm{M}-\mathrm{CH}_{2} \mathrm{NO}_{2}\right]^{+}\right)$.

HRMS (ESI $\left.{ }^{+}\right): m / z[\mathrm{M}+\mathrm{Na}]^{+}$calcd for $\mathrm{C}_{22} \mathrm{H}_{21} \mathrm{NO}_{4} \mathrm{Na}$ : 386.1363; found: 386.1351.

\section{(R)-(Z)-6,6-Dimethyl-2-(naphthalen-1-ylmethylene)-3-(nitro- methyl)-3,5,6,7-tetrahydrobenzofuran-4(2H)-one (3h)}

Compound $\mathbf{3 h}$ was isolated after flash chromatography (n-pentane/ $\left.\mathrm{Et}_{2} \mathrm{O}, 1: 1\right)$; yield: $82 \mathrm{mg}(90 \%)$; colorless solid; $\mathrm{mp} 165-167^{\circ} \mathrm{C}$; $R_{f}=0.23$ ( $n$-pentane/ $\left.\mathrm{Et}_{2} \mathrm{O}, 1: 1\right) ;[\alpha]_{\mathrm{D}}{ }^{24}+110.1$ ( $c=0.4$, benzene).

HPLC: Daicel Chiralpak IB, $n$-heptane/EtOH (7:3), $0.5 \mathrm{~mL} / \mathrm{min}, \lambda=230$ $\mathrm{nm}, t_{\mathrm{R}}($ minor $)=15.9 \mathrm{~min}, t_{\mathrm{R}}$ (major) $=16.9 \mathrm{~min} ; 95 \%$ ee .

IR (ATR): 3056, 2959, 1648, 1550, 1398, 1294, 1218, 1172, 1141, 1089 , $1019,975,915,838,780,699 \mathrm{~cm}^{-1}$.

${ }^{1} \mathrm{H}$ NMR $\left(600 \mathrm{MHz}, \mathrm{CDCl}_{3}\right): \delta=7.98(\mathrm{~d}, J=8.3 \mathrm{~Hz}, 1 \mathrm{H}, \mathrm{ArH}), 7.87-7.77$ $(\mathrm{m}, 3 \mathrm{H}, \mathrm{ArH}), 7.56-7.46(\mathrm{~m}, 3 \mathrm{H}, \mathrm{ArH}), 6.41(\mathrm{~d}, J=2.1 \mathrm{~Hz}, 1 \mathrm{H}, \mathrm{OC}=\mathrm{CH})$, $5.02\left(\mathrm{dd}, J=13.1,3.9 \mathrm{~Hz}, 1 \mathrm{H}, \mathrm{CHHNO}_{2}\right), 4.83(\mathrm{dd}, J=13.1,7.2 \mathrm{~Hz}, 1 \mathrm{H}$, $\left.\mathrm{CHHNO}_{2}\right), 4.72-4.66\left(\mathrm{~m}, 1 \mathrm{H}, \mathrm{CHCH}_{2}\right), 2.48\left(\mathrm{~m}, 2 \mathrm{H}, \mathrm{CH}_{2}\right), 2.32(\mathrm{~m}, 2 \mathrm{H}$, $\left.\mathrm{CH}_{2}\right), 1.16$ (s, $\left.3 \mathrm{H}, \mathrm{CH}_{3}\right), 1.15\left(\mathrm{~s}, 3 \mathrm{H}, \mathrm{CH}_{3}\right)$.

${ }^{13} \mathrm{C}$ NMR $\left(151 \mathrm{MHz}, \mathrm{CDCl}_{3}\right): \delta=193.6(\mathrm{C}=0), 175.2\left(\mathrm{C}_{\mathrm{q}}\right), 154.2\left(\mathrm{C}_{\mathrm{q}}\right)$, 133.6 $\left(C_{q}\right), 131.1\left(C_{q}\right), 129.4\left(C_{q}\right), 128.6(\operatorname{ArC}), 128.1(\operatorname{ArC}), 127.1(\operatorname{ArC})$, 126.4 (ArC), $125.9(\operatorname{ArC}), 125.3(\operatorname{ArC}), 123.8(\operatorname{ArC}), 111.5\left(C_{\mathrm{q}}\right), 103.5$ $(\mathrm{OC}=\mathrm{CH}), 76.0\left(\mathrm{CH}_{2} \mathrm{NO}_{2}\right), 51.0\left(\mathrm{CH}_{2}\right), 41.7\left(\mathrm{CHCH}_{2}\right), 37.2\left(\mathrm{CH}_{2}\right), 34.4$ $\left[\mathrm{C}\left(\mathrm{CH}_{3}\right)_{2}\right], 29.0\left(\mathrm{CH}_{3}\right), 28.3\left(\mathrm{CH}_{3}\right)$.

MS (EI, $70 \mathrm{eV}): m / z(\%)=364.4\left(2,[\mathrm{M}+\mathrm{H}]^{+}\right), 363.3\left(7,[\mathrm{M}]^{+}\right), 317.3(24$, $\left.\left[\mathrm{M}-\mathrm{NO}_{2}\right]^{+}\right), 303.3\left(6,\left[\mathrm{M}-\mathrm{CH}_{2} \mathrm{NO}_{2}\right]^{+}\right)$.

HRMS (ESI $\left.{ }^{+}\right): m / z[\mathrm{M}+\mathrm{Na}]^{+}$calcd for $\mathrm{C}_{22} \mathrm{H}_{21} \mathrm{NO}_{4} \mathrm{Na}$ : 386.1323; found: 386.1345 .

\section{(R)-(Z)-2-(Furan-2-ylmethylene)-6,6-dimethyl-3-(nitromethyl)-} 3,5,6,7-tetrahydrobenzofuran-4(2H)-one (3i)

Compound $3 \mathbf{i}$ was isolated after flash chromatography (n-pentane/ $\left.\mathrm{Et}_{2} \mathrm{O}, 1: 1\right)$; yield: $73 \mathrm{mg}(96 \%)$; colorless solid; $\mathrm{mp} 103-105^{\circ} \mathrm{C}$; $R_{f}=0.31$ ( $n$-pentane/ $\left.\mathrm{Et}_{2} \mathrm{O}, 1: 1\right) ;[\alpha]_{D}{ }^{24}+85.7$ ( $c=0.4$, benzene).

HPLC: Daicel Chiralpak IB, $n$-heptane/EtOH (7:3), $0.7 \mathrm{~mL} / \mathrm{min}, \lambda=254$ $\mathrm{nm}, t_{\mathrm{R}}($ minor $)=9.3 \mathrm{~min}, t_{\mathrm{R}}$ (major) $=10.3 \mathrm{~min} ; 96 \%$ ee .

IR (ATR): 2960, 2876, 2081, 1988, 1649, 1554, 1466, 1374, 1292, 1228 , $1167,1089,1049,1016,989,920,884,816,747,700,671 \mathrm{~cm}^{-1}$.
${ }^{1} \mathrm{H}$ NMR $\left(600 \mathrm{MHz}, \mathrm{C}_{6} \mathrm{D}_{6}\right): \delta=7.05(\mathrm{~d}, J=1.6 \mathrm{~Hz}, 1 \mathrm{H}, \mathrm{OCH}), 6.59(\mathrm{~d}, J=$ $3.3 \mathrm{~Hz}, 1 \mathrm{H}, \mathrm{CH}), 6.19$ (dd, J = 3.3, $1.8 \mathrm{~Hz}, 1 \mathrm{H}, \mathrm{CH}), 5.61(\mathrm{~d}, J=2.3 \mathrm{~Hz}, 1$ $\mathrm{H}, \mathrm{OC}=\mathrm{CH}), 4.29$ (dd, $\left.J=13.2,6.1 \mathrm{~Hz}, 1 \mathrm{H}, \mathrm{CHHNO}_{2}\right), 4.04(\mathrm{dd}, J=13.2$, $\left.3.7 \mathrm{~Hz}, 1 \mathrm{H}, \mathrm{CHHNO}_{2}\right), 3.79\left(\mathrm{~s}, 1 \mathrm{H}, \mathrm{CHCH}_{2}\right), 1.90(\mathrm{~d}, J=16.0 \mathrm{~Hz}, 1 \mathrm{H}$, $\left.\mathrm{CH}_{2}\right), 1.82\left(\mathrm{~d}, J=16.0 \mathrm{~Hz}, 1 \mathrm{H}, \mathrm{CH}_{2}\right), 1.71\left(\mathrm{~d}, J=17.8 \mathrm{~Hz}, 1 \mathrm{H}, \mathrm{CH}_{2}\right), 1.63$ (d, J = $\left.17.8 \mathrm{~Hz}, 1 \mathrm{H}, \mathrm{CH}_{2}\right), 0.62\left(\mathrm{~s}, 3 \mathrm{H}, \mathrm{CH}_{3}\right), 0.57\left(\mathrm{~s}, 3 \mathrm{H}, \mathrm{CH}_{3}\right)$.

${ }^{13} \mathrm{C}$ NMR (151 MHz, $\left.\mathrm{C}_{6} \mathrm{D}_{6}\right): \delta=191.6(\mathrm{C}=0), 173.2\left(\mathrm{C}_{\mathrm{q}}\right), 152.1\left(\mathrm{C}_{\mathrm{q}}\right)$, $149.1\left(\mathrm{C}_{\mathrm{q}}\right), 141.2(\mathrm{OCH}), 111.6(\mathrm{CH}), 111.3\left(\mathrm{C}_{\mathrm{q}}\right), 109.5(\mathrm{CH}), 96.2$ $(\mathrm{OC}=\mathrm{CH}), 74.8\left(\mathrm{CH}_{2} \mathrm{NO}_{2}\right), 50.5\left(\mathrm{CH}_{2}\right), 41.4\left(\mathrm{CHCH}_{2}\right), 36.2\left(\mathrm{CH}_{2}\right), 33.2$ $\left[\mathrm{C}\left(\mathrm{CH}_{3}\right)_{2}\right], 28.3\left(\mathrm{CH}_{3}\right), 27.2\left(\mathrm{CH}_{3}\right)$.

MS (EI, $70 \mathrm{eV}): m / z(\%)=304.1\left(1,[\mathrm{M}+\mathrm{H}]^{+}\right), 303.1\left(5,[\mathrm{M}]^{+}\right), 257.1(24$, $\left.\left[\mathrm{M}-\mathrm{NO}_{2}\right]^{+}\right), 243.0\left(13,\left[\mathrm{M}-\mathrm{CH}_{2} \mathrm{NO}_{2}\right]^{+}\right)$.

HRMS $\left(\mathrm{ESI}^{+}\right): \mathrm{m} / z[\mathrm{M}+\mathrm{Na}]^{+}$calcd for $\mathrm{C}_{16} \mathrm{H}_{17} \mathrm{NO}_{5} \mathrm{Na}$ : 326.0999; found: 326.0990 .

\section{(R)-(Z)-2-Benzylidene-3-(nitromethyl)-3,5,6,7-tetrahydrobenzo-} furan-4(2H)-one (5a)

Compound 5a was isolated after flash chromatography (n-pentane/Et $\left.{ }_{2} \mathrm{O}, 1: 2\right)$; yield: $56 \mathrm{mg}(79 \%)$; colorless solid; mp $117-119{ }^{\circ} \mathrm{C}$; $R_{f}=0.14$ ( $n$-pentane/Et $\left.{ }_{2} \mathrm{O}, 1: 1\right) ;[\alpha]_{D}{ }^{24}+74.9$ ( $c=0.5$, benzene).

HPLC: Daicel Chiralpak AS, $n$-heptane/EtOH (7:3), $0.7 \mathrm{~mL} / \mathrm{min}, \lambda=254$ $\mathrm{nm}, t_{\mathrm{R}}($ minor $)=13.3 \mathrm{~min}, t_{\mathrm{R}}$ (major) $=15.9 \mathrm{~min} ; 98 \%$ ee .

IR (ATR): 2953, 2322, 2087, 1892, 1641, 1547, 1384, 1217, 1176, 1051 , $974,841,696 \mathrm{~cm}^{-1}$.

${ }^{1} \mathrm{H}$ NMR $\left(600 \mathrm{MHz}, \mathrm{CDCl}_{3}\right): \delta=7.54(\mathrm{~d}, J=7.4 \mathrm{~Hz}, 2 \mathrm{H}, \mathrm{ArH}), 7.34(\mathrm{t}, J=$ $7.8 \mathrm{~Hz}, 2 \mathrm{H}, \operatorname{ArH}), 7.24(\mathrm{t}, J=7.4 \mathrm{~Hz}, 1 \mathrm{H}, \operatorname{ArH}), 5.73(\mathrm{~d}, J=2.1 \mathrm{~Hz}, 1 \mathrm{H}$, $\mathrm{OC}=\mathrm{CH}), 4.92\left(\mathrm{dd}, J=13.2,3.8 \mathrm{~Hz}, 1 \mathrm{H}, \mathrm{CHHNO}_{2}\right), 4.67(\mathrm{dd}, J=13.2,7.6$ $\left.\mathrm{Hz}, 1 \mathrm{H}, \mathrm{CHHNO}_{2}\right), 4.58\left(\mathrm{~m}, 1 \mathrm{H}, \mathrm{CHCH}_{2}\right), 2.72-2.65\left(\mathrm{~m}, 2 \mathrm{H}, \mathrm{CH}_{2}\right)$, 2.47-2.42 (m, $2 \mathrm{H}, \mathrm{CH}_{2}$ ), 2.16 (qi, J $=6.4 \mathrm{~Hz}, 2 \mathrm{H}, \mathrm{CH}_{2}$ ).

${ }^{13} \mathrm{C}$ NMR (151 MHz, $\left.\mathrm{CDCl}_{3}\right): \delta=194.1(\mathrm{C}=\mathrm{O}), 176.0\left(\mathrm{C}_{\mathrm{q}}\right), 152.8\left(\mathrm{C}_{\mathrm{q}}\right)$, $133.3\left(\mathrm{C}_{\mathrm{q}}\right), 128.7(\operatorname{ArC}), 128.5(\operatorname{ArC}), 127.3(\operatorname{ArC}), 112.7\left(\mathrm{C}_{\mathrm{q}}\right), 106.8$ $(\mathrm{OC}=\mathrm{CH}), 75.9\left(\mathrm{CH}_{2} \mathrm{NO}_{2}\right), 41.7\left(\mathrm{CHCH}_{2}\right), 36.6\left(\mathrm{CH}_{2}\right), 23.4\left(\mathrm{CH}_{2}\right), 21.5$ $\left(\mathrm{CH}_{2}\right)$.

MS (EI, $70 \mathrm{eV}): m / z(\%)=286.3\left(13,[\mathrm{M}+\mathrm{H}]^{+}\right), 285.2\left(2,[\mathrm{M}]^{+}\right), 239.3$ $\left(21,\left[\mathrm{M}-\mathrm{NO}_{2}\right]^{+}\right), 225.2\left(19,\left[\mathrm{M}-\mathrm{CH}_{2} \mathrm{NO}_{2}\right]^{+}\right)$.

HRMS (ESI $\left.{ }^{+}\right): m / z[\mathrm{M}+\mathrm{Na}]^{+}$calcd for $\mathrm{C}_{16} \mathrm{H}_{15} \mathrm{NO}_{4} \mathrm{Na}$ : 308.0893; found: 308.0893 .

\section{(R)-4-(Nitromethyl)-2-phenyl-6,7-dihydrocyclopenta[b]pyran- 5(4H)-one (5b)}

Compound $\mathbf{5 b}$ was isolated after flash chromatography (n-pentane/Et $\left.{ }_{2} \mathrm{O}, 1: 2\right)$; yield: $43 \mathrm{mg}(63 \%)$; colorless solid; $\mathrm{mp} 153-155^{\circ} \mathrm{C}$; $R_{f}=0.19$ ( $n$-pentane/Et $\left.{ }_{2} \mathrm{O}, 1: 1\right) ;[\alpha]_{\mathrm{D}}{ }^{24}-162.9$ ( $c=0.4$, benzene).

HPLC: Daicel Chiralpak IC, $n$-heptane/EtOH (7:3), $0.7 \mathrm{~mL} / \mathrm{min}, \lambda=230$ $\mathrm{nm}, t_{\mathrm{R}}($ minor $)=10.7 \mathrm{~min}, t_{\mathrm{R}}$ (major) $=8.8 \mathrm{~min}$; $94 \%$ ee .

IR (ATR): 3075, 2922, 2308, 2096, 1902, 1665, 1543, 1393, 1234, 991, $856,764,696 \mathrm{~cm}^{-1}$.

${ }^{1} \mathrm{H}$ NMR $\left(600 \mathrm{MHz}, \mathrm{C}_{6} \mathrm{D}_{6}\right): \delta=7.32(\mathrm{~m}, 2 \mathrm{H}, \mathrm{ArH}), 7.04(\mathrm{~m}, 3 \mathrm{H}, \mathrm{ArH})$, $5.09(\mathrm{~d}, J=3.6 \mathrm{~Hz}, 1 \mathrm{H}, \mathrm{OC}=\mathrm{CH}), 4.20\left(\mathrm{~m}, 2 \mathrm{H}, \mathrm{CH}_{2} \mathrm{NO}_{2}\right), 3.47(\mathrm{~m}, 1 \mathrm{H}$, $\mathrm{CHCH}_{2}$ ), 1.89 (ddd, $\left.J=17.8,7.4,2.8 \mathrm{~Hz}, 1 \mathrm{H}, \mathrm{CH}\right), 1.81$ (ddd, $J=17.9$, 7.4, $2.5 \mathrm{~Hz}, 1 \mathrm{H}, \mathrm{CH}), 1.75(\mathrm{~m}, 1 \mathrm{H}, \mathrm{CH}), 1.68(\mathrm{~m}, 1 \mathrm{H}, \mathrm{CH})$.

${ }^{13} \mathrm{C}$ NMR (151 MHz, $\left.\mathrm{C}_{6} \mathrm{D}_{6}\right): \delta=200.7(\mathrm{C}=0), 179.2\left(\mathrm{C}_{\mathrm{q}}\right), 150.7\left(\mathrm{C}_{\mathrm{q}}\right)$, $132.3\left(\mathrm{C}_{\mathrm{q}}\right), 129.2(\operatorname{ArC}), 128.4(2 \mathrm{C}, \operatorname{ArC}), 124.9(2 \mathrm{C}, \operatorname{ArC}), 111.8\left(\mathrm{C}_{\mathrm{q}}\right)$, 98.7 $(\mathrm{OC}=\mathrm{CH}), 76.6\left(\mathrm{CH}_{2} \mathrm{NO}_{2}\right), 32.8\left(\mathrm{CH}_{2}\right), 29.8\left(\mathrm{CHCH}_{2}\right), 24.9\left(\mathrm{CH}_{2}\right)$.

MS (EI, $70 \mathrm{eV}): m / z(\%)=272.2\left(3,[\mathrm{M}+\mathrm{H}]^{+}\right), 225.2\left(27,\left[\mathrm{M}-\mathrm{NO}_{2}\right]^{+}\right)$, $211.2\left(84,\left[\mathrm{M}-\mathrm{CH}_{2} \mathrm{NO}_{2}\right]^{+}\right)$. 
HRMS (ESI $\left.{ }^{+}\right): m / z[\mathrm{M}+\mathrm{Na}]^{+}$calcd for $\mathrm{C}_{15} \mathrm{H}_{13} \mathrm{NO}_{4} \mathrm{Na}$ : 294.0737; found: 294.0732 .

\section{(R)-4-(Nitromethyl)-2-phenyl-4,8-dihydropyrano[3,4-b]pyran- $5(6 H)$-one (5c)}

Compound 5c was isolated after flash chromatography (n-pentane/Et ${ }_{2} \mathrm{O}, 1: 1$ to $\left.1: 2\right)$; yield: $22 \mathrm{mg}(31 \%)$; colorless solid; mp 113$115^{\circ} \mathrm{C} ; R_{f}=0.43(n$-pentane/Et $20,1: 1) ;[\alpha]_{D}{ }^{24}-213.5(c=0.4$, benzene).

HPLC: Daicel Chiralpak IA, $n$-heptane/i-PrOH (7:3), $0.7 \mathrm{~mL} / \mathrm{min}, \lambda=$ $254 \mathrm{~nm}, t_{\mathrm{R}}$ (minor) $=14.2 \mathrm{~min}, t_{\mathrm{R}}$ (major) $=11.9 \mathrm{~min}$; $90 \%$ ee.

IR (ATR): 2962, 1675, 1635, 1546, 1494, 1439, 1393, 1270, 1229, 1139, 1056, 994, 952, 873, 814, 764, $677 \mathrm{~cm}^{-1}$.

${ }^{1} \mathrm{H}$ NMR $\left(600 \mathrm{MHz}, \mathrm{C}_{6} \mathrm{D}_{6}\right): \delta=7.22-7.19(\mathrm{~m}, 2 \mathrm{H}, \mathrm{ArH}), 7.05-7.02(\mathrm{~m}, 3$ $\mathrm{H}, \mathrm{ArH}), 5.10(\mathrm{~d}, J=4.3 \mathrm{~Hz}, 1 \mathrm{H}, \mathrm{OC}=\mathrm{CH}), 4.06(\mathrm{dd}, J=11.7,7.0 \mathrm{~Hz}, 1 \mathrm{H}$, $\left.\mathrm{CHHNO}_{2}\right), 4.01\left(\mathrm{dd}, J=11.7,3.8 \mathrm{~Hz}, 1 \mathrm{H}, \mathrm{CHHNO}{ }_{2}\right), 3.92(\mathrm{~d}, J=15.9 \mathrm{~Hz}$, $\left.1 \mathrm{H}, \mathrm{CH}_{2}\right), 3.75\left(\mathrm{~d}, J=16.2 \mathrm{~Hz}, 1 \mathrm{H}, \mathrm{CH}_{2}\right), 3.67-3.63\left(\mathrm{~m}, 1 \mathrm{H}, \mathrm{CHCH}_{2}\right)$, 3.59 (dd, $\left.J=15.9,1.7 \mathrm{~Hz}, 1 \mathrm{H}, \mathrm{CH}_{2}\right), 3.52\left(\mathrm{dd}, J=16.1,1.4 \mathrm{~Hz}, 1 \mathrm{H}, \mathrm{CH}_{2}\right)$. ${ }^{13} \mathrm{C}$ NMR (151 MHz, $\left.\mathrm{C}_{6} \mathrm{D}_{6}\right): \delta=192.1(\mathrm{C}=\mathrm{O}), 165.3\left(\mathrm{C}_{\mathrm{q}}\right), 149.2\left(\mathrm{C}_{\mathrm{q}}\right)$, $131.9\left(\mathrm{C}_{\mathrm{q}}\right), 129.2(\operatorname{ArC}), 128.3(\operatorname{ArC}), 124.6(\operatorname{ArC}), 106.1\left(\mathrm{C}_{\mathrm{q}}\right), 99.1$ $(\mathrm{OC}=\mathrm{CH}), 77.6\left(\mathrm{CH}_{2} \mathrm{NO}_{2}\right), 71.1\left(\mathrm{CH}_{2}\right), 63.5\left(\mathrm{CH}_{2}\right), 28.4\left(\mathrm{CHCH}_{2}\right)$.

MS (EI, $70 \mathrm{eV}): m / z(\%)=241.1\left(17,\left[\mathrm{M}-\mathrm{NO}_{2}\right]^{+}\right), 227.1(45,[\mathrm{M}-$ $\left.\mathrm{CH}_{2} \mathrm{NO}_{2}\right]^{+}$.

HRMS (ESI $\left.{ }^{+}\right): m / z[\mathrm{M}+\mathrm{Na}]^{+}$calcd for $\mathrm{C}_{15} \mathrm{H}_{13} \mathrm{NO}_{5} \mathrm{Na}$ : 310.0686; found: 310.0686 .

\section{(R)-1,3-Dimethyl-5-(nitromethyl)-7-phenyl-1,5-dihydro-2H-pyra-} no[2,3-d]pyrimidine-2,4(3H)-dione (5d)

Compound 5d was isolated after flash chromatography [n-pentane $/ \mathrm{CH}_{2} \mathrm{Cl}_{2}$ (1:4) to pure $\mathrm{CH}_{2} \mathrm{Cl}_{2}$ ]; yield: $77 \mathrm{mg}$ (94\%); bright yellow solid; $\mathrm{mp} 224-226^{\circ} \mathrm{C} ; R_{f}=0.26\left(\mathrm{CH}_{2} \mathrm{Cl}_{2} / \mathrm{Et}_{2} \mathrm{O}, 20: 1\right) ;[\alpha]_{\mathrm{D}}{ }^{24}-144.5(c=$ $0.4, \mathrm{CHCl}_{3}$ ).

HPLC: Daicel Chiralpak AS, $n$-heptane/EtOH (7:3), $1.0 \mathrm{~mL} / \mathrm{min}, \lambda=254$ $\mathrm{nm}, t_{\mathrm{R}}($ minor $)=10.3 \mathrm{~min}, t_{\mathrm{R}}$ (major) $=7.5 \mathrm{~min} ; 88 \%$ ee .

IR (ATR): 2954, 2324, 2107, 1645, 1476, 1375, 1203, 1003, 753, 690 $\mathrm{cm}^{-1}$.

${ }^{1} \mathrm{H} \mathrm{NMR}\left(600 \mathrm{MHz}, \mathrm{CDCl}_{3}\right): \delta=7.58-7.52(\mathrm{~m}, 2 \mathrm{H}, \mathrm{ArH}), 7.45-7.41(\mathrm{~m}$, $3 \mathrm{H}, \mathrm{ArH}), 5.71(\mathrm{~d}, J=4.4 \mathrm{~Hz}, 1 \mathrm{H}, \mathrm{OC}=\mathrm{CH}), 4.83-4.74\left(\mathrm{~m}, 2 \mathrm{H}, \mathrm{CH}_{2} \mathrm{NO}_{2}\right)$, $4.24\left(\mathrm{dt}, J=6.1,4.3 \mathrm{~Hz}, 1 \mathrm{H}, \mathrm{CHCH}_{2}\right), 3.54\left(\mathrm{~s}, 3 \mathrm{H}, \mathrm{CH}_{3}\right), 3.39(\mathrm{~s}, 3 \mathrm{H}$, $\mathrm{CH}_{3}$ ).

${ }^{13} \mathrm{C}$ NMR (151 MHz, $\left.\mathrm{CDCl}_{3}\right): \delta=162.0(\mathrm{C}=0), 154.3(\mathrm{C}=0), 150.5\left(\mathrm{C}_{\mathrm{q}}\right)$, $149.7\left(C_{q}\right), 131.2\left(C_{q}\right), 130.0(\operatorname{ArC}), 128.8(2 \mathrm{C}, \operatorname{ArC}), 124.8$ (2 C, $\left.\operatorname{ArC}\right)$, $99.2(\mathrm{OC}=\mathrm{CH}), 84.0\left(\mathrm{C}_{\mathrm{q}}\right), 77.9\left(\mathrm{CH}_{2} \mathrm{NO}_{2}\right), 30.9\left(\mathrm{CHCH}_{2}\right), 29.2\left(\mathrm{CH}_{3}\right), 28.2$ $\left(\mathrm{CH}_{3}\right)$.

MS (EI, $70 \mathrm{eV}) m / z(\%)=283.1\left(20,\left[\mathrm{M}-\mathrm{NO}_{2}\right]^{+}\right), 269.0(71,[\mathrm{M}-$ $\left.\mathrm{CH}_{2} \mathrm{NO}_{2}\right]^{+}$.

HRMS (ESI ${ }^{+}$): $m / z$ [M $\left.+\mathrm{Na}\right]^{+}$calcd for $\mathrm{C}_{16} \mathrm{H}_{15} \mathrm{~N}_{3} \mathrm{O}_{5} \mathrm{Na}$ : 352.0904; found: 352.0894 .

(R)-(Z)-2-Benzylidene-6-methyl-3-(nitromethyl)-3,5,6,7-tetrahydrobenzofuran-4(2H)-one (7a)

Compound 7a was isolated after flash chromatography (n-pentane/ $\mathrm{Et}_{2} \mathrm{O}, 1: 1$ to $1: 2$ ); yield: $73 \mathrm{mg}(98 \%)$; colorless solid; mp 175$177^{\circ} \mathrm{C} ; R_{f}=0.24$ (n-pentane/Et $\left.2 \mathrm{O}, 1: 1\right) ;[\alpha]_{\mathrm{D}}{ }^{24}+85.5(c=0.5, \mathrm{MeOH})$.
HPLC: Daicel Chiralpak IC, $n$-heptane/ $i$-PrOH (7:3), $0.5 \mathrm{~mL} / \mathrm{min}, \lambda=$ $254 \mathrm{~nm}, t_{\mathrm{R}}$ (minor) $1=27.5 \mathrm{~min}, t_{\mathrm{R}}$ (major) $1=16.6 \mathrm{~min} ; t_{\mathrm{R}}($ minor $) 2=$ $29.9 \mathrm{~min}, t_{\mathrm{R}}$ (major) $2=18.2 \mathrm{~min} ; 94 \%$ ee, $\mathrm{dr}=1: 1$.

IR (ATR): 2951, 2925, 2871, 2153, 2086, 2048, 1989, 1735, 1691, 1650, 1549, 1389, 1288, 1205, 1163, 1099, 1016, 973, 914, 873, 847, 811, $752,694 \mathrm{~cm}^{-1}$.

${ }^{1} \mathrm{H}$ NMR $\left(600 \mathrm{MHz}, \mathrm{C}_{6} \mathrm{D}_{6}\right): \delta=7.51\left(\mathrm{~m}, 4 \mathrm{H}, \operatorname{ArH}, \operatorname{ArH}_{\text {Diast. }}\right), 7.18(\mathrm{~m}, 4 \mathrm{H}$, Ar-H, $\left.\operatorname{ArH}_{\text {Diast. }}\right), 7.03$ (m, $\left.2 \mathrm{H}, \mathrm{ArH}, \mathrm{ArH}_{\text {Diast. }}\right), 5.44$ (d, $J=2.2 \mathrm{~Hz}, 1 \mathrm{H}$, $\mathrm{OC}=\mathrm{CH}), 5.42\left(\mathrm{~d}, J=2.2 \mathrm{~Hz}, 1 \mathrm{H}, \mathrm{OC}=\mathrm{CH}_{\text {Diast. }}\right), 4.34(\mathrm{dd}, J=13.1,6.4 \mathrm{~Hz}$, $1 \mathrm{H}, \mathrm{CH}_{2} \mathrm{NO}_{2}$ ), 4.28 (dd, $J=13.0,6.6 \mathrm{~Hz}, 1 \mathrm{H}, \mathrm{CH}_{2} \mathrm{NO}_{2}$ Diast.), 4.22 (ddd, $J=$ 13.0, 7.9, $3.8 \mathrm{~Hz}, 2 \mathrm{H}, \mathrm{CH}_{2} \mathrm{NO}_{2}, \mathrm{CH}_{2} \mathrm{NO}_{2}$ Diast.), $3.94\left(\mathrm{~m}, 2 \mathrm{H}, \mathrm{CHCH}_{2}\right.$, $\mathrm{CHCH}_{2}$ Diast.), 2.07 ( $\mathrm{m}, 4 \mathrm{H}, \mathrm{CH}_{2}, \mathrm{CH}_{2}$ Diast.), 1.65 ( $\mathrm{m}, 4 \mathrm{H}, \mathrm{CH}_{2}, \mathrm{CH}_{2}$ Diast.), $1.56\left(\mathrm{~m}, 2 \mathrm{H}, \mathrm{CHCH}_{3}, \mathrm{CHCH}_{3}\right.$ Diast. $), 1.44(\mathrm{~m}, 1 \mathrm{H}, \mathrm{CHH}), 1.27(\mathrm{~m}, 1 \mathrm{H}$, $\left.\mathrm{CHH}_{\text {Diast. }}\right), 0.50$ (d, $\left.J=6.6 \mathrm{~Hz}, 3 \mathrm{H}, \mathrm{CH}_{3}\right), 0.46(\mathrm{~d}, J=6.3 \mathrm{~Hz}, 3 \mathrm{H}$, $\mathrm{CH}_{3}$ Diast.).

${ }^{13} \mathrm{C}$ NMR $\left(151 \mathrm{MHz}, \mathrm{C}_{6} \mathrm{D}_{6}\right): \delta=191.8(\mathrm{C}=\mathrm{O}), 191.8\left(\mathrm{C}=\mathrm{O}_{\text {Diast. }}\right), 174.2\left(\mathrm{C}_{\mathrm{q}}\right)$, $174.0\left(\mathrm{C}_{\mathrm{q} \text { Diast. }}\right), 153.3\left(\mathrm{C}_{\mathrm{q}}\right), 153.3\left(\mathrm{C}_{\mathrm{q} \text { Diast. }}\right), 133.9\left(\mathrm{C}_{\mathrm{q}}\right), 133.9\left(\mathrm{C}_{\mathrm{q} \text { Diast. }}\right)$, 128.7 ( 4 C, $\operatorname{ArC}, \operatorname{ArC}_{\text {Diast. }}$ ), 128.4 ( 4 C, $\operatorname{ArC}, \operatorname{ArC}_{\text {Diast. }}$ ), 127.0 (2 C, ArC, $\operatorname{ArC}$. Diast. $), 112.1\left(\mathrm{C}_{\mathrm{q}}\right), 111.9\left(\mathrm{C}_{\mathrm{q} \text { Diast. }}\right), 106.1(\mathrm{OC}=\mathrm{CH}), 106.0\left(\mathrm{OC}=\mathrm{CH}_{\text {Diast. }}\right)$, $75.7\left(\mathrm{CH}_{2} \mathrm{NO}_{2}\right), 75.1\left(\mathrm{CH}_{2} \mathrm{NO}_{2}\right.$ Diast. $), 44.6\left(\mathrm{CH}_{2}\right), 44.5\left(\mathrm{CH}_{2}\right.$ Diast. $), 41.9$ $\left(\mathrm{CHCH}_{2}\right), 41.8\left(\mathrm{CHCH}_{2}\right.$ Diast. $), 30.3\left(\mathrm{CH}_{2}\right), 30.2\left(\mathrm{CH}_{2}\right.$ Diast. $), 29.2\left(\mathrm{CHCH}_{3}\right)$, $29.0\left(\mathrm{CHCH}_{3 \text { Diast. }}\right), 20.1\left(\mathrm{CH}_{3}\right), 12.0\left(\mathrm{CH}_{3 \text { Diast. }}\right)$.

MS (EI, $70 \mathrm{eV}): m / z(\%)=300.0\left(3,[\mathrm{M}+\mathrm{H}]^{+}\right), 253.1\left(19,\left[\mathrm{M}-\mathrm{NO}_{2}\right]^{+}\right)$, $239.0\left(11,\left[\mathrm{M}-\mathrm{CH}_{2} \mathrm{NO}_{2}\right]^{+}\right)$.

HRMS (ESI $\left.{ }^{+}\right): m / z[\mathrm{M}+\mathrm{Na}]^{+}$calcd for $\mathrm{C}_{17} \mathrm{H}_{17} \mathrm{NO}_{4} \mathrm{Na}$ : 322.1050 ; found: 322.1049.

\section{$(R)$-(Z)-2-Benzylidene-6-isopropyl-3-(nitromethyl)-3,5,6,7-tetra- hydrobenzofuran- $4(2 \mathrm{H})$-one $(7 \mathrm{~b})$}

Compound $\mathbf{7 b}$ was isolated after flash chromatography (n-pentane/ $\mathrm{Et}_{2} \mathrm{O}, 1: 1$ to $\left.1: 2\right)$; yield: $67 \mathrm{mg}(82 \%)$; colorless solid; $\mathrm{mp} 141-$ $143^{\circ} \mathrm{C} ; R_{f}=0.33$ ( $n$-pentane/Et $\left.2 \mathrm{O}, 1: 1\right) ;[\alpha]_{\mathrm{D}}{ }^{24}+52.7$ ( $c=0.5$, benzene). HPLC: Daicel Chiralpak IA, $n$-heptane/EtOH (97:3), $1.0 \mathrm{~mL} / \mathrm{min}, \lambda=$ $254 \mathrm{~nm}, t_{\mathrm{R}}$ (minor) $1=41.7 \mathrm{~min}, t_{\mathrm{R}}$ (major) $1=63.8 \mathrm{~min} ; t_{\mathrm{R}}$ (minor) $2=$ $50.4 \mathrm{~min}, t_{\mathrm{R}}$ (major) $2=58.3 \mathrm{~min} ; 93 \% e e, \mathrm{dr}=1: 1$.

IR (ATR): 3352, 2956, 2325, 2096, 1649, 1546, 1381, 1100, 958, 842, $756,687 \mathrm{~cm}^{-1}$.

${ }^{1} \mathrm{H}$ NMR $\left(600 \mathrm{MHz}, \mathrm{C}_{6} \mathrm{D}_{6}\right): \delta=7.53\left(\mathrm{~m}, 4 \mathrm{H}, \mathrm{ArH}, \operatorname{ArH}_{\text {Diast. }}\right), 7.20$ (m, 4 $\left.\mathrm{H}, \mathrm{ArH}, \operatorname{ArH}_{\text {Diast. }}\right), 7.05$ (m, $\left.2 \mathrm{H}, \mathrm{ArH}, \operatorname{ArH}_{\text {Diast. }}\right), 5.49(\mathrm{~d}, J=2.1 \mathrm{~Hz}, 1 \mathrm{H}$, $\mathrm{OC}=\mathrm{CH}), 5.47\left(\mathrm{~d}, J=2.1 \mathrm{~Hz}, 1 \mathrm{H}, \mathrm{OC}=\mathrm{CH}_{\text {Diast. }}\right), 4.35\left(\mathrm{~m}, 1 \mathrm{H}, \mathrm{CH} \mathrm{HNO}_{2}\right)$, $4.30\left(\mathrm{~m}, 3 \mathrm{H}, \mathrm{CH}_{2} \mathrm{NO}_{2}, \mathrm{CH}_{2} \mathrm{NO}_{2}\right.$ Diast. $), 4.05\left(\mathrm{~m}, 1 \mathrm{H}, \mathrm{CHCH}_{2}\right), 4.01(\mathrm{~m}, 1 \mathrm{H}$, $\left.\mathrm{CHCH}_{2 \text { Diast. }}\right), 2.17$ ( $\left.\mathrm{m}, 2 \mathrm{H}, \mathrm{CHH}, \mathrm{CHH}_{\text {Diast. }}\right), 1.79\left(\mathrm{~m}, 3 \mathrm{H}, \mathrm{CHH}, \mathrm{CHH}_{\text {Diast. }}\right)$, $1.59\left[\mathrm{~m}, 2 \mathrm{H}, \mathrm{CHCH}\left(\mathrm{CH}_{3}\right)_{2}, \mathrm{CHCH}\left(\mathrm{CH}_{3}\right)_{2}\right.$ Diast. ], $1.43(\mathrm{~m}, 2 \mathrm{H}, \mathrm{CHH}, \mathrm{CHH}$ Diast.), 1.31 ( $\mathrm{m}, 1 \mathrm{H}, \mathrm{CHH}), 1.01$ [ $\mathrm{m}, 2 \mathrm{H}, \mathrm{CH}\left(\mathrm{CH}_{3}\right)_{2}, \mathrm{CH}\left(\mathrm{CH}_{3}\right)_{2}$ Diast.], 0.48 $\left[\mathrm{m}, 12 \mathrm{H}, \mathrm{CH}\left(\mathrm{CH}_{3}\right)_{2}, \mathrm{CH}\left(\mathrm{CH}_{3}\right)_{2}\right.$ Diast. $]$.

${ }^{13} \mathrm{C}$ NMR (151 MHz, $\left.\mathrm{C}_{6} \mathrm{D}_{6}\right): \delta=192.1(\mathrm{C}=\mathrm{O}), 192.1(\mathrm{C}=\mathrm{O}$ Diast. $), 174.7$ $\left(\mathrm{C}_{\mathrm{q}}\right), 174.7\left(\mathrm{C}_{\mathrm{q} \text { Diast. }}\right), 153.4\left(\mathrm{C}_{\mathrm{q}}\right), 153.4\left(\mathrm{C}_{\mathrm{q} \text { Diast }}\right), 133.9\left(\mathrm{C}_{\mathrm{q}}\right), 133.9\left(\mathrm{C}_{\mathrm{q} \text { Diast. }}\right)$, 128.7 ( 4 C, $\operatorname{ArC}, \operatorname{ArC}_{\text {Diast. }}$ ), 128.4 ( 4 C, $\operatorname{ArC}, \operatorname{ArC}_{\text {Diast. }}$ ), 127.1 (2 C, $\operatorname{ArC}, \operatorname{ArC}$ Diast. $), 112.2\left(\mathrm{C}_{\mathrm{q}}\right), 112.0\left(\mathrm{C}_{\mathrm{q} \text { Diast. }}\right), 106.1(\mathrm{OC}=\mathrm{CH}), 106.0\left(\mathrm{OC}=\mathrm{CH}_{\text {Diast. }}\right)$, $75.8\left(\mathrm{CH}_{2} \mathrm{NO}_{2}\right), 75.1\left(\mathrm{CH}_{2} \mathrm{NO}_{2}\right.$ Diast. $), 41.9\left(\mathrm{CHCH}_{2}\right), 41.9\left(\mathrm{CHCH}_{2}\right.$ Diast. $)$, $40.7\left(\mathrm{CH}_{2}\right), 40.4\left(\mathrm{CH}_{2}\right.$ Diast. $), 40.3\left[\mathrm{CHCH}\left(\mathrm{CH}_{3}\right)_{2}\right], 40.2\left[\mathrm{CHCH}\left(\mathrm{CH}_{3}\right)_{2}\right.$ Diast. $]$, $31.4\left[\mathrm{CH}\left(\mathrm{CH}_{3}\right)_{2}\right], 31.3\left[\mathrm{CH}\left(\mathrm{CH}_{3}\right)_{2}\right.$ Diast. $], 26.1\left(\mathrm{CH}_{2}\right), 25.9\left(\mathrm{CH}_{2}\right.$ Diast. $), 19.3$ $\left(\mathrm{CH}_{3}\right), 19.1\left(\mathrm{CH}_{3}\right.$ Diast. $), 18.8\left(\mathrm{CH}_{3}\right), 18.7\left(\mathrm{CH}_{3}\right.$ Diast. $)$.

MS $\left(\mathrm{EI}^{+}, 70 \mathrm{eV}\right): m / z(\%)=328.1\left(3,[\mathrm{M}+\mathrm{H}]^{+}\right), 327.1\left(1,[\mathrm{M}]^{+}\right), 281.1$ (33, $\left.\left[\mathrm{M}-\mathrm{NO}_{2}\right]^{+}\right), 267.1\left(8,\left[\mathrm{M}-\mathrm{CH}_{2} \mathrm{NO}_{2}\right]^{+}\right)$.

HRMS (ESI $\left.{ }^{+}\right): m / z$ [M $\left.+\mathrm{Na}\right]^{+}$calcd for $\mathrm{C}_{19} \mathrm{H}_{21} \mathrm{NO}_{4} \mathrm{Na}$ : 350.1363; found: 350.1362 . 
(R)-(Z)-2-Benzylidene-3-(nitromethyl)-6-phenyl-3,5,6,7-tetrahydrobenzofuran- $4(2 \mathrm{H})$-one $(7 \mathrm{c})$

Compound 7c was isolated after flash chromatography (n-pentane/ $\mathrm{Et}_{2} \mathrm{O}, 1: 1$ to $1: 2$ ); yield: $85 \mathrm{mg}(94 \%)$; colorless solid; mp $128-$ $130{ }^{\circ} \mathrm{C} ; R_{f}=0.24$ ( $n$-pentane/ $\left.\mathrm{Et}_{2} \mathrm{O}, 1: 1\right) ;[\alpha]_{\mathrm{D}}{ }^{24}+64.8(c=0.5$, benzene). HPLC: Daicel Chiralpak AD, $n$-heptane/EtOH (1:1), $1.0 \mathrm{~mL} / \mathrm{min}, \lambda=$ $254 \mathrm{~nm}, t_{\mathrm{R}}$ (minor) $1=9.2 \mathrm{~min}, t_{\mathrm{R}}$ (major) $1=17.1 \mathrm{~min} ; t_{\mathrm{R}}$ (minor) $2=$ $11.0 \mathrm{~min}, t_{\mathrm{R}}$ (major) $2=13.9 \mathrm{~min} ; 94 \%$ ee, $\mathrm{dr}=1: 1$.

IR (ATR): 3027, 2305, 2102, 1910, 1735, 1647, 1546, 1397, 1204, 1002 , $909,853,753,694 \mathrm{~cm}^{-1}$.

${ }^{1} \mathrm{H} \mathrm{NMR}\left(600 \mathrm{MHz}, \mathrm{C}_{6} \mathrm{D}_{6}\right): \delta=7.51\left(\mathrm{~m}, 4 \mathrm{H}, \mathrm{ArH}, \mathrm{ArH}_{\text {Diast. }}\right), 7.19(\mathrm{~m}, 4 \mathrm{H}$, $\left.\mathrm{ArH}, \mathrm{ArH}_{\text {Diast. }}\right), 7.04$ (m, $8 \mathrm{H}, \mathrm{ArH}$, Diast.), $6.72\left(\mathrm{~m}, 4 \mathrm{H}, \mathrm{ArH}, \mathrm{ArH}_{\text {Diast. }}\right)$, $5.48\left(\mathrm{~s}, 2 \mathrm{H}, \mathrm{OC}=\mathrm{CH}, \mathrm{OC}=\mathrm{CH}_{\text {Diast. }}\right), 4.32\left(\mathrm{~m}, 4 \mathrm{H}, \mathrm{CH}_{2} \mathrm{NO}_{2}, \mathrm{CH}_{2} \mathrm{NO}_{2}\right.$ Diast. $)$, $4.06\left(\mathrm{~m}, 1 \mathrm{H}, \mathrm{CHCH}_{2}\right), 4.00\left(\mathrm{~m}, 1 \mathrm{H}, \mathrm{CHCH}_{2}\right.$ Diast. $), 2.83\left(\mathrm{~m}, 1 \mathrm{H}, \mathrm{CHC}_{6} \mathrm{H}_{5}\right)$, $2.70\left(\mathrm{~m}, 1 \mathrm{H}, \mathrm{CHC}_{6} \mathrm{H}_{5 \text { Diast. }}\right), 2.36\left(\mathrm{~m}, 2 \mathrm{H}, \mathrm{CH}_{2}\right.$ Diast.), 2.23 (dd, $J=16.2$, $12.5 \mathrm{~Hz}, 1 \mathrm{H}, \mathrm{CHH}), 2.11$ (dd, J = 16.2, $12.9 \mathrm{~Hz}, 1 \mathrm{H}, \mathrm{CHH}), 1.92(\mathrm{~m}, 4 \mathrm{H}$, $\mathrm{CH}_{2}, \mathrm{CH}_{2}$ Diast.).

${ }^{13} \mathrm{C}$ NMR (151 MHz, $\left.\mathrm{C}_{6} \mathrm{D}_{6}\right): \delta=191.0(\mathrm{C}=\mathrm{O}), 190.9\left(\mathrm{C}=\mathrm{O}_{\text {Diast. }}\right), 173.8\left(\mathrm{C}_{\mathrm{q}}\right)$, $173.8\left(\mathrm{C}_{\mathrm{q} \text { Diast. }}\right), 153.3\left(\mathrm{C}_{\mathrm{q}}\right), 153.2\left(\mathrm{C}_{\mathrm{q} \text { Diast }}\right), 142.0\left(\mathrm{C}_{\mathrm{q}}\right), 142.0\left(\mathrm{C}_{\mathrm{q} \text { Diast. }}\right)$, $133.8\left(\mathrm{C}_{\mathrm{q}}\right), 133.8$ ( $\mathrm{C}_{\mathrm{q} \text { Diast. }}$ ), 128.7 (4 C, $\left.\operatorname{ArC}, \operatorname{ArC}_{\text {Diast. }}\right), 128.5$ (4 C, $\operatorname{ArC}$, $\left.\operatorname{ArC}_{\text {Diast. }}\right), 128.4$ (4 C, $\left.\operatorname{ArC}, \operatorname{ArC}_{\text {Diast. }}\right), 127.1$ (2 C, $\left.\operatorname{ArC}, \operatorname{ArC}_{\text {Diast. }}\right), 127.0$ (2 C, $\left.\operatorname{ArC}, \operatorname{ArC}_{\text {Diast. }}\right), 126.6$ (4 C, $\left.\operatorname{ArC}, \operatorname{ArC}_{\text {Diast. }}\right) 112.3\left(C_{\mathrm{q}}\right), 112.2\left(\mathrm{C}_{\mathrm{q} \text { Diast. }}\right), 106.3$ $(\mathrm{OC}=\mathrm{CH}), 106.3\left(\mathrm{OC}=\mathrm{CH}_{\text {Diast. }}\right), 75.7\left(\mathrm{CH}_{2} \mathrm{NO}_{2}\right), 75.0\left(\mathrm{CH}_{2} \mathrm{NO}_{2}\right.$ Diast. $), 43.7$ $\left(\mathrm{CH}_{2}\right), 43.6\left(\mathrm{CH}_{2}\right.$ Diast. $), 41.9\left(\mathrm{CHCH}_{2}\right), 41.8\left(\mathrm{CHCH}_{2}\right.$ Diast. $), 39.7(\mathrm{CHPh})$, $39.6\left(\mathrm{CHPh}_{\text {Diast. }}\right), 30.1\left(\mathrm{CH}_{2}\right), 30.0\left(\mathrm{CH}_{2}\right.$ Diast. $)$.

MS $\left(\mathrm{EI}^{+}, 70 \mathrm{eV}\right): m / z(\%)=361.1\left(1,[\mathrm{M}+\mathrm{H}]^{+}\right), 315.1\left(38,\left[\mathrm{M}-\mathrm{NO}_{2}\right]^{+}\right)$, $301.1\left(7,\left[\mathrm{M}-\mathrm{CH}_{2} \mathrm{NO}_{2}\right]^{+}\right)$.

HRMS (ESI $\left.{ }^{+}\right): m / z[\mathrm{M}+\mathrm{Na}]^{+}$calcd for $\mathrm{C}_{22} \mathrm{H}_{19} \mathrm{NO}_{4} \mathrm{Na}$ : 384.1206; found: 384.1207.

\section{(R)-(Z)-2-Benzylidene-6-(furan-2-yl)-3-(nitromethyl)-3,5,6,7-tet-} rahydrobenzofuran $-4(2 \mathrm{H})$-one $(7 \mathrm{~d})$

Compound 7d was isolated after flash chromatography (n-pentane/Et $\left.{ }_{2} \mathrm{O}, 1: 1\right)$; yield: $86 \mathrm{mg}(98 \%)$; colorless solid; mp $143-145^{\circ} \mathrm{C}$; $R_{f}=0.21$ (n-pentane/Et $\left.2 \mathrm{O}, 1: 1\right) ;[\alpha]_{\mathrm{D}}{ }^{24}+64.8(c=0.4, \mathrm{MeOH})$.

SFC: Daicel Chiralcel OJ-H, CO $/ \mathrm{MeCN}(8: 2), 4.0 \mathrm{~mL} / \mathrm{min}, \lambda=222 \mathrm{~nm}, t_{\mathrm{R}}$ (minor) $1=3.9 \mathrm{~min}, t_{\mathrm{R}}$ (major) $1=5.2 \mathrm{~min} ; t_{\mathrm{R}}$ (minor) $2=7.1 \mathrm{~min}, t_{\mathrm{R}}$ (major) $2=9.7 \mathrm{~min} ; 99 \%$ ee, $\mathrm{dr}=1: 1$.

IR (ATR): 2914, 2327, 2098, 1647, 1547, 1393, 1201, 1009, 849, 752 $\mathrm{cm}^{-1}$.

${ }^{1} \mathrm{H}$ NMR (600 MHz, $\left.\mathrm{C}_{6} \mathrm{D}_{6}\right): \delta=7.47$ (t, $J=6.9 \mathrm{~Hz}, 4 \mathrm{H}, \mathrm{ArH}, \mathrm{ArH}_{\text {Diast. }}$ ), 7.17 (m, $\left.4 \mathrm{H}, \mathrm{ArH}, \mathrm{ArH}_{\text {Diast. }}\right), 7.03$ (m, $\left.2 \mathrm{H}, \mathrm{ArH}, \mathrm{ArH}_{\text {Diast. }}\right), 6.96-6.93$ (m, $\left.2 \mathrm{H}, \mathrm{OCH}, \mathrm{OCH}_{\text {Diast. }}\right), 5.97$ (ddd, $J=6.6,3.2,1.9 \mathrm{~Hz}, 2 \mathrm{H}, \mathrm{ArH}, \mathrm{ArH}_{\text {Diast. }}$ ), $5.65\left(\mathrm{~d}, J=3.2 \mathrm{~Hz}, 2 \mathrm{H}, \mathrm{ArH}, \mathrm{ArH}_{\text {Diast. }}\right), 5.44(\mathrm{~d}, J=2.2 \mathrm{~Hz}, 1 \mathrm{H}, \mathrm{OC}=\mathrm{CH})$, $5.41\left(\mathrm{~d}, J=2.2 \mathrm{~Hz}, 1 \mathrm{H}, \mathrm{OC}=\mathrm{CH}_{\text {Diast. }}\right), 4.25\left(\mathrm{~m}, 2 \mathrm{H}, \mathrm{CHHNO}_{2}, \mathrm{CHHNO}_{2}\right.$ Diast.), 4.17 (m, $2 \mathrm{H}, \mathrm{CHHNO}_{2}, \mathrm{CHHNO}_{2}$ Diast.), 4.01 ( $\left.\mathrm{m}, 1 \mathrm{H}, \mathrm{CHCH}_{2}\right), 3.89$ $\left(\mathrm{m}, 1 \mathrm{H}, \mathrm{CHCH}_{2 \text { Diast. }}\right), 2.88(\mathrm{~m}, 1 \mathrm{H}, \mathrm{CHAr}), 2.82\left(\mathrm{~m}, 1 \mathrm{H}, \mathrm{CHAr}_{\text {Diast. }}\right), 2.37$ ( $\mathrm{m}, 2 \mathrm{H}, \mathrm{CH}_{2}, \mathrm{CH}_{2}$ Diast.), 2.21 ( $\mathrm{m}, 2 \mathrm{H}, \mathrm{CH}_{2}, \mathrm{CH}_{2}$ Diast.), 2.07 ( $\mathrm{m}, 4 \mathrm{H}, \mathrm{CH}_{2}$, $\mathrm{CH}_{2 \text { Diast. }}$ ).

${ }^{13} \mathrm{C}$ NMR (151 MHz, $\left.\mathrm{C}_{6} \mathrm{D}_{6}\right): \delta=190.2\left(2 \mathrm{C}, \mathrm{C}=\mathrm{O}, \mathrm{C}=\mathrm{O}_{\text {Diast. }}\right), 172.9\left(\mathrm{C}_{\mathrm{q}}\right)$, $172.8\left(\mathrm{C}_{\mathrm{q} \text { Diast. }}\right), 155.1\left(\mathrm{C}_{\mathrm{q}}\right), 155.0\left(\mathrm{C}_{\mathrm{q} \text { Diast. }}\right), 153.1\left(\mathrm{C}_{\mathrm{q}}\right), 153.0\left(\mathrm{C}_{\mathrm{q} \text { Diast. }}\right)$, $141.4\left(2 \mathrm{C}, \mathrm{OCH}_{\text {furanyl }}, \mathrm{OCH}_{\text {furanyl, Diast. }}\right), 133.7\left(\mathrm{C}_{\mathrm{q}}\right), 133.7\left(\mathrm{C}_{\mathrm{q} \text { Diast. }}\right), 128.7$ (4 C, $\left.\operatorname{ArC}, \operatorname{ArC}_{\text {Diast. }}\right), 128.3$ ( 4 C, $\operatorname{ArC}, \operatorname{ArC}_{\text {Diast. }}$ ), 127.1 (2 C, $\operatorname{ArC}, \operatorname{ArC}_{\text {Diast. }}$ ), $112.3\left(\mathrm{C}_{\mathrm{q}}\right), 112.2\left(\mathrm{C}_{\mathrm{q} \text { Diast. }}\right), 110.1\left(\mathrm{CH}_{\text {furanyl }}\right), 110.1\left(\mathrm{CH}_{\text {furanyl, Diast. }}\right), 106.3$ $(\mathrm{OC}=\mathrm{CH}), 106.3(\mathrm{OC}=\mathrm{CO}$ Diast. $), 104.9\left(\mathrm{CH}_{\text {furanyl }}\right), 104.8\left(\mathrm{CH}_{\text {furanyl, Diast. }}\right)$, $75.4\left(\mathrm{CH}_{2} \mathrm{NO}_{2}\right), 75.0\left(\mathrm{CH}_{2} \mathrm{NO}_{2}\right.$ Diast. $), 41.8\left(\mathrm{CHCH}_{2}\right), 41.7\left(\mathrm{CHCH}_{2}\right.$ Diast. $)$, $40.8\left(\mathrm{CH}_{2}\right), 40.7\left(\mathrm{CH}_{2}\right.$ Diast. $), 33.0\left(\mathrm{CH}_{\text {furanyl }}\right), 32.8\left(\mathrm{CH}_{\text {furanyl, Diast. }}\right), 27.4(2$ $\mathrm{C}, \mathrm{CH}_{2}, \mathrm{CH}_{2}$ Diast.).
MS (EI, $70 \mathrm{eV}): m / z(\%)=351.1\left(1,[\mathrm{M}]^{+}\right), 305.1\left(44,\left[\mathrm{M}-\mathrm{NO}_{2}\right]^{+}\right), 291.1$ (7, $\left.\left[\mathrm{M}-\mathrm{CH}_{2} \mathrm{NO}_{2}\right]^{+}\right)$.

HRMS $\left(\mathrm{ESI}^{+}\right): m / z[\mathrm{M}+\mathrm{Na}]^{+}$calcd for $\mathrm{C}_{20} \mathrm{H}_{17} \mathrm{NO}_{5} \mathrm{Na}$ : 374.0999; found: 374.0997.

\section{Acknowledgment}

Financial support from the European Research Council (ERC Advanced Grant 320493 (DOMINOCAT') is gratefully acknowledged. We thank Prof. Englert, Institute of Inorganic Chemistry for his help with the Xray crystal structure determination of $\mathbf{5 a}$.

\section{Supporting Information}

Supporting information for this article is available online at http://dx.doi.org/10.1055/s-0035-1561468.

\section{References}

(1) (a) Sainsbury, M. In Heterocyclic Chemistry; Abel, E. W., Ed.; The Royal Society of Chemistry: Cambridge, 2001, 58. (b) Wolfe, J. P.; Hay, M. B. Tetrahedron 2007, 63, 261. (c) Miyabe, H.; Miyata, O.; Naito, T. In Heterocycles in Natural Product Synthesis; Majumdar, K. C.; Chattopadhyay, S. K., Eds.; Wiley-VCH: Weinheim, 2011, 153. (d) Lorente, A.; Lamariano-Merketegi, J.; Albericio, F.; Álvarez, M. Chem. Rev. 2013, 113, 4567. (e) Dar, A. M.; Shamsuzzaman, Eur. Chem. Bull. 2015, 4, 249. (f) Kumar, K. A.; Renuka, N.; Kumar, G. V.; Lokeshwari, D. M. J. Chem. Pharm. Res. 2015, 7, 693.

(2) (a) Juo, R.-R.; Herz, W. J. Org. Chem. 1985, 50, 700. (b) Srikrishna, A.; Krishnan, K. Tetrahedron Lett. 1988, 29, 4995. (c) Tanrisever, N.; Fischer, N. H.; Williamson, G. B. Phytochemistry 1988, 27, 2523. (d) Aso, M.; Qjida, A.; Yang, G.; Cha, O.-J.; Osawa, E.; Kanematsu, K. J. Org. Chem. 1993, 58, 3960. (e) Lee, Y. R.; Lee, G. J.; Kang, K. Y. Bull. Korean Chem. Soc. 2002, 23, 1477.

(3) Joshi, S. C.; Mathela, C. S. Pharmacog. Res. 2012, 4, 80.

(4) Jung, H. W.; Mahesh, R.; Park, J. H.; Boo, Y. C.; Park, K. M.; Park, Y.-K. Int. Immunopharmacol. 2010, 10, 155.

(5) (a) Gong, J.; Lin, G.; Sun, W.; Li, C.-C.; Yang, Z. J. Am. Chem. Soc. 2010, 132, 16745. (b) Lu, P.; Mailyan, A.; Gu, Z.; Guptill, D. M.; Wang, H.; Davies, H. M. L.; Zakarian, A. J. Am. Chem. Soc. 2014, 136, 17738. (c) Zheng, C.; Dubovyk, I.; Lazarski, K. E.; Thomson, R. J. J. Am. Chem. Soc. 2014, 136, 17750. (d) Zhang, W.-B.; Lin, G.; Shao, W.-B.; Gong, J.-X.; Yang, Z. Chem. Asian J. 2015, 10, 903. (e) Zhang, W.-B.; Shao, W.-B.; Li, F.-Z.; Gong, J.-X.; Yang, Z. Chem. Asian J. 2015, 10, 1874.

(6) (a) Adili, A.; Tao, Z.-L.; Chen, D.-F.; Han, Z.-Y. Org. Biomol. Chem. 2015, 13, 2247. (b) Wu, B.; Gao, X.; Yan, Z.; Huang, W.-X.; Zhou, Y.-G. Tetrahedron Lett. 2015, 56, 4334.

(7) (a) Barange, D. K.; Raju, B. R.; Kavala, V.; Kuo, C.-W.; Tu, Y.-C.; Yao, C.-F. Tetrahedron 2010, 66, 3754. (b) Han, Y.; Hou, H.; Yao, R.; Fu, Q.; Yan, C.-G. Synthesis 2010, 4061. (c) Rueping, M.; Parra, A.; Uria, U.; Besselièvre, F.; Merino, E. Org. Lett. 2010, 12, 5680. (d) Devi, R. B.; Henrot, M.; De Paolis, M.; Maddaluno, J. Org. Biomol. Chem. 2011, 9, 6509. (e) Dong, L.; Deng, L.; Lim, Y. H.; Leung, G. Y. C.; Chen, D. Y.-K. Chem. Eur.J. 2011, 17, 5778. (f) Wu, M.-Y.; Wang, M.-Q.; Li, K.; Feng, X.-W.; He, T.; Wang, N.; Yu, W.Q. Tetrahedron Lett. 2011, 52, 679. (g) Chawla, R.; Singh, A. K.; Yadav, L. D. S. Tetrahedron Lett. 2012, 53, 3382. (h) Jonek, A.; Berger, S.; Haak, E. Chem. Eur. J. 2012, 18, 15504. (i) Liu, Z.; Fan, 
G.-P.; Wang, G.-W. Chem. Commun. 2012, 48, 11665. (j) Kalpogiannaki, D.; Martini, C.-I.; Nikopoulou, A.; Nyxas, J. A.; Pantazi, V.; Hadjiarapoglou, L. P. Tetrahedron 2013, 69, 1566. (k) Xia, L.; Lee, Y. R. Adv. Synth. Catal. 2013, 355, 1261. (l) Yao, C.; Wang, Y.; Li, T.; Yu, C.; Li, L.; Wang, C. Tetrahedron 2013, 69, 10593. (m) Kasare, S.; Bankar, S. K.; Ramasastry, S. S. V. Org. Lett. 2014, 16, 4284. (n) Bosnidou, A.-E.; Kalpogiannaki, D.; Karanestora, S.; Nixas, J. A.; Hadjiarapoglou, L. P. J. Org. Chem. 2015, 80, 1279. (o) Riveira, M. J.; Quiroga, G. N.; Mata, E. G.; Gandon, V.; Mischne, M. P. J. Org. Chem. 2015, 80, 6515. (p) Wang, S.; He, L.-Y.; Guo, L.-N. Synthesis 2015, 47, 3191. (q) Wei, J.; Nie, B.-J.; Peng, R.; Cheng, X.-H.; Wang, S.; He, P. Synlett 2016, 27, 626. (r) Kale, A.; Chennapuram, M.; Bingi, C.; Nanubolu, J. B.; Atmakur, K. Org. Biomol. Chem. 2016, 14, 582. (s) Liu, W.; Lai, X.; Zha, G.; Xu, Y.; Sun, P.; Xia, T.; Shen, Y. Org. Biomol. Chem. 2016, 14, 3603.

(8) Sinha, D.; Biswas, A.; Singh, V. K. Org. Lett. 2015, 17, 3302.

(9) Feng, J.; Lin, L.; Yu, K.; Liu, X.; Feng, X. Adv. Synth. Catal. 2015, 357, 1305.

(10) Calter, M. A.; Korotkov, A. Org. Lett. 2015, 17, 1385.

(11) El-Sepelgy, O.; Haseloff, S.; Alamsetti, S. K.; Schneider, C. Angew. Chem. Int. Ed. 2014, 53, 7923.

(12) Kumar, R. K.; Bi, X. Chem. Commun. 2016, 52, 853.

(13) (a) Zhang, L.; Fang, G.; Kumar, R. K.; Bi, X. Synthesis 2015, 47, 2317. (b) Zi, W.; Toste, D. Chem. Soc. Rev. 2016, 45, in press; DOI: 10.1039/c5cs00929d. (c) Yoshida, H. ACS Catal. 2016, 6, 1799.

(14) (a) Naodovic, M.; Yamamoto, H. Chem. Rev. 2008, 108, 3132. (b) Yamamoto, Y. Chem. Rev. 2008, 108, 3199. (c) Weibel, J.-M.; Blanc, A.; Pale, P. Chem. Rev. 2008, 108, 3149. (d) Álvarez-Corral, M.; Munoz-Dorado, M.; Rodríguez-García, I. Chem. Rev. 2008, 108, 3174. (e) Belmont, P.; Parker, E. Eur. J. Org. Chem. 2009, 6075. (f) Fang, G.; Bi, X. Chem. Soc. Rev. 2015, 44, 8124. (g) Sekine, K.; Yamada, T. Chem. Soc. Rev. 2016, 45, in press; DOI: $10.1039 / \mathrm{c} 5 \mathrm{cs} 00895 \mathrm{f}$.
(15) For examples of merging organo- and transition metal-catalysis, see: (a) Ding, Q.; Wu, J. Org. Lett. 2007, 9, 4959. (b) Shao, Z.; Zhang, H. Chem. Soc. Rev. 2009, 38, 2745. (c) Zhong, C.; Shi, X. Eur. J. Org. Chem. 2010, 2999. (d) Arróniz, C.; Gil-González, A.; Semak, V.; Escolano, C.; Bosch, J.; Amat, M. Eur. J. Org. Chem. 2011, 3755. (e) Loh, C. C. J.; Enders, D. Chem. Eur. J. 2012, 18, 10212. (f) Du, Z.; Shao, Z. Chem. Soc. Rev. 2013, 42, 1337. (g) Deng, Y.; Kumar, S.; Wang, H. Chem. Commun. 2014, 50, 4272.

(16) (a) Hack, D.; Loh, C. C. J.; Hartmann, J. M.; Raabe, G.; Enders, D. Chem. Eur. J. 2014, 20, 3917. (b) Hack, D.; Chauhan, P.; Deckers, K.; Hermann, G. N.; Mertens, L.; Raabe, G.; Enders, D. Org. Lett. 2014, 16, 5188. (c) Hack, D.; Chauhan, P.; Deckers, K.; Mizutani, Y.; Raabe, G.; Enders, D. Chem. Commun. 2015, 51, 2266. (d) Hack, D.; Dürr, A. B.; Deckers, K.; Chauhan, P.; Seling, N.; Rübenach, L.; Mertens, L.; Raabe, G.; Schoenebeck, F.; Enders, D. Angew. Chem. Int. Ed. 2016, 53, 1797. (e) Kaya, U.; Chauchan, P.; Hack, D.; Deckers, K.; Puttreddy, R.; Rissanen, K.; Enders, D. Chem. Commun. 2016, 52, 1669.

(17) (a) Alemán, J.; Parra, A.; Jiang, H.; Jørgensen, K. A. Chem. Eur. J. 2011, 17, 6890. (b) Storer, R. I.; Aciro, C.; Jones, L. H. Chem. Soc. Rev. 2011, 40, 2330. (c) Ni, X.; Li, X.; Wang, Z.; Cheng, J.-P. Org. Lett. 2014, 16, 1786. (d) Chauhan, P.; Mahajan, S.; Kaya, U.; Hack, D.; Enders, D. Adv. Synth. Catal. 2015, 357, 253.

(18) CCDC 1474771 (5a) and CCDC 1474975 (5d) contain the supplementary crystallographic data for this paper. The data can be obtained free of charge from The Cambridge Crystallographic Data Centre via www.ccdc.cam.ac.uk/getstructures.

(19) Yang, W.; Du, D.-M. Adv. Synth. Catal. 2011, 353, 1241.

(20) (a) Malerich, J. P.; Hagihara, K.; Rawal, V. H. J. Am. Chem. Soc. 2008, 130, 14416. (b) Zhu, Y.; Malerich, J. P.; Rawal, V. H. Angew. Chem. Int. Ed. 2010, 49, 153. 